\title{
ANOTHER HOMOGENEOUS PLANE CONTINUUM
}

\author{
BY \\ R. H. BING AND F. B. JONES
}

1. History of problem. A set $X$ is homogeneous if for each pair of points $x, y$ of $X$ there is a homeomorphism of $X$ onto itself that takes $x$ into $y$.

In 1920 Knaster and Kuratowski [21] raised the following question: If a nondegenerate bounded plane continuum is homogeneous, is it necessarily a simple closed curve?

In 1922, Knaster [20] described a hereditarily indecomposable plane continuum. It is reported that he suspected that this continuum had other interesting properties and suggested to his students the problem of discovering if this Knaster continuum (as it came to be called) had the property possessed by an arc of being topologically equivalent to each of its nondegenerate subcontinua. This Knaster continuum is homogeneous and furnishes a counterexample to an affirmative answer of the above question, but this was not discovered until 1951.

A partial affirmative solution was given to the question in 1924 when Mazurkiewicz [22] showed that the bounded nondegenerate homogeneous plane continuum is a simple closed curve if it is locally connected. This result was improved in 1951 when Cohen [12] showed that the continuum is a simple closed curve if it either is arcwise connected or contains a simple closed curve.

A false affirmative solution was announced [25] in 1937. (Of course, at the time, it was not known that the solution was false-this only developed eleven years later when a counter-example was given.) This false solution was extended [11] in 1944 when an attempt was made to classify all homogeneous bounded closed plane sets.

That a pseudo-arc is homogeneous was shown first by Bing [3] in 1948 and shortly thereafter by essentially the same methods by Moise [24]. Both of these proofs made use of the description of the pseudo-arc given by Moise [23] to show that a pseudo-arc is topologically equivalent to each of its nondegenerate subcontinua.

In 1951 Bing [4] discovered that the pseudo-arc described by Moise in 1948 is actually topologically equivalent to the continuum Knaster described twenty-six years earlier by different methods. In fact, it was shown that any two nondegenerate snake-like hereditarily indecomposable continua are topologically equivalent. Also, it was shown that in the category sense, most

Presented to the Society, September 2, 1954 by R. H. Bing and by F. B. Jones under the title On a certain type of homogeneous plane continuum; received by the editors April 15, 1957. 
bounded continua in $E^{n}(n>1)$ are pseudo-arcs. Hence, most bounded plane continua are homogeneous.

In 1953 two papers $[18 ; 19]$ appeared questioning the homogeneity of the pseudo-arc. The second of the papers made an unsuccessful attempt to correct an error in the first. Inasmuch as these papers received not unfavorable reviews (Mathematical Reviews vol. 15 (1954) p. 146; p. 335) despite the errors in them, it seems desirable to mention that the pseudo-arc has not been abandoned as an example of a homogeneous plane continuum.

In 1954, working independently, Bing and Jones each discovered a homogeneous plane continuum that was neither a simple closed curve nor a pseudo-arc. Neither knew of the others work until the titles of the papers appeared adjacent to each other on the 1954 summer program of the American Mathematical Society $[6 ; 15]$. Inasmuch as both had discovered the same example, it was decided to make this a joint paper. The first part of this paper showing that the example - a circle of pseudo-arcs - is homogeneous was written by Bing. The latter part showing that such a circle of pseudo-arcs can be imbedded in the plane was prepared by Jones.

Perhaps the future holds the answer as to whether or not there are other homogeneous bounded plane continua. Jones' result [14] that each bounded homogeneous plane continuum which does not separate the plane is indecomposable may guide our search. Bing has described a continuum (Example 2 of [4]) that is suspected of being homogeneous. However, at the moment, the only nondegenerate bounded plane continua known to be homogeneous are the simple closed curve, the pseudo-arc, and the circle of pseudo-arcs described in this paper. Other pertinent references are found in the bibliography of this paper.

2. The example. In this section we describe a homogeneous plane continuum which we call a circle of pseudo-arcs. First we define some terms that we shall use.

A chain is a finite collection $D$ of open sets $d_{1}, d_{2}, \cdots, d_{n}$ such that $d_{i}$ intersects $d_{j}$ if $i, j$ are adjacent integers and otherwise $\rho\left(d_{i}, d_{j}\right)$ is positive. We say that $i, j$ are adjacent if $|i-j| \leqq 1$-hence $i$ is adjacent to itself. We use $\rho$ to denote the distance function. In earlier papers the weaker condition that nonadjacent links did not intersect was used instead of the stronger condition that they were a positive distance apart but in some respects this is less convenient for our present purposes than the stronger condition.

The elements $d_{1}, d_{n}$ of the above chain $D$ are called end links of $D$ and the other links are called interior links. If each link is of diameter less than $\epsilon$, $D$ is called an $\epsilon$-chain. The subchain of $D$ consisting of $d_{r}, d_{r+1}, \cdots, d_{s}$ is denoted by $D(r, s)$.

Suppose $D=d_{1}, d_{2}, \cdots, d_{n}$ is a chain which covers a point set $X$. Then each point of $X$ lies in some link of $D$. We say that $D$ properly covers $X$ if each link of $D$ contains a point of $X$. Also, $D$ irreducibly covers $X$ of each link 
of $D$ contains a point of $X$ that is not covered by any other link of $D$. If a chain irreducibly covers a set, then it properly covers the set, but not conversely.

A compact continuum is called snake-like [5] if for each positive number $\epsilon$, it can be covered by an $\epsilon$-chain. The pseudo-arc described in $[23 ; 3 ; 4 ; 7]$ is an example of a snake-like continuum.

A continuum $G$ is called an arc of continua $\left\{g_{x} \mid 0 \leqq x \leqq 1\right\}$ if there is a map $f$ of $G$ onto $[0,1]$ such that $f^{-1}(x)$ is the continuum $g_{x}$. Then $\left\{g_{x}\right\}$ is an upper semicontinuous collection of continua filling $G$ such that the corresponding decomposition space is an arc. Then $g_{0}$ and $g_{1}$ are called the end elements of $\left\{g_{x}\right\}$. We use $G(a, b)$ to denote the sum of all continua in the collection $\left\{g_{x} \mid a \leqq x \leqq b\right\}$.

Such a continuum $G$ is a snake-like arc of continua $\left\{g_{x} \mid 0 \leqq x \leqq 1\right\}$ if it is snake-like. If each element of $\left\{g_{x}\right\}$ is a pseudo-arc, $G$ is called a snake-like arc of pseudo-arcs. If in addition, the collection $\left\{g_{x}\right\}$ is continuous ( $f$ is open), then $G$ is called a continuous snake-like arc of pseudo-arcs. In Theorem 10 we show that any two continuous snake-like arcs of pseudo-arcs of homeomorphic-indeed there is a very strong type of homeomorphism between them.

A circular chain differs from a regular chain in that the first and last links intersect. A compact nonsnake-like continuum which for each positive number $\epsilon$ can be covered by an $\epsilon$-circular chain is called circle-like.

The bounded plane continuum which is shown in this paper to be homogeneous is a continuous circle-like circle of pseudo-arcs-which we call for brevity, a circle of pseudo-arcs. It is a circle-like continuum $M$ such that there is a continuous decomposition of $M$ into pseudo-arcs such that the decomposition space is a simple closed curve. Hence, there is an open map $f$ of $M$ onto a circle such that the inverse of each point of the circle is a pseudo-arc.

Perhaps certain of the continua used by Anderson in $[1 ; 2]$ were circles of pseudo-arcs but this was not pointed out there. It follows from Theorem 10 that any two circles of pseudo-arcs are topologically equivalent and that each is homogeneous.

In establishing homeomorphisms between snake-like continua it is frequently convenient to consider sequences of chains covering them and relations between these chains. The chain $D_{2}$ refines the chain $D_{1}$ if each link of $D_{2}$ lies in a link of $D_{1}$. A chain map $H$ of a chain $E$ into a chain $D$ is a single valued function that assigns a link of $D$ to each link of $E$ such that the images of adjacent links are adjacent-that is $H\left(e_{i}\right)$ and $H\left(e_{i+1}\right)$ are adjacent links of $D$. If each link of $D$ is the image of a link of $E$, we say that $H$ maps $E$ onto $D$. We note that the chain map $H$ does not operate on the points in the links of $E$ but only on the links. Chain maps play much the same role in this paper as following a pattern did in [3].

3. Snake-like arcs of continua. In this section we prove some theorems 
about snake-like continua. A modification of the argument in the following proof shows that each tree-like continuum as defined in [5] is unicoherent but we shall not be concerned with tree-like continua in this paper.

THEOREM 1. Each snake-like continuum is unicoherent.

Proof. Suppose a snake-like continuum $M$ is the sum of two continua $M_{1}, M_{2}$. We show that $M$ is unicoherent by showing that each pair of points $p, q$ of $M_{1} \cdot M_{2}$ belongs to a component in $M_{1} \cdot M_{2}$.

Suppose $d_{1}, d_{2}, \cdots, d_{n}$ is a chain covering $M, p \in d_{i}, q \in d_{j}$, and $i<j$. Since each of $M_{1}, M_{2}$ is connected and contains $p+q$, each intersects each link of $d_{i}, d_{i+1}, \cdots, d_{j}$. Hence there is a sequence of points $p=p_{i}, p_{i+1}, \cdots, p_{j}$ $=q$ where $p_{r} \in d_{r} \cdot M_{1}$. If $d_{1}, d_{2}, \cdots, d_{n}$ is an $\epsilon$-chain, then $\rho\left(p_{r}, M_{2}\right)<\epsilon$ and $\rho\left(p_{r}, p_{r+1}\right)<2 \epsilon$.

Since $M$ is snake-like, for each positive number $\epsilon$ it can be covered by an $\epsilon$-chain. The results of the preceding paragraph show that there is a sequence $R_{1}, R_{2}, \cdots$ such that $R_{k}$ is a finite number of points $p=p_{1}^{k}$, $p_{2}^{k}, \cdots, p_{t}^{k}=q$ such that $p_{r}^{k} \in M_{1}, \rho\left(p_{r}^{k}, M_{2}\right)<1 / k$, and $\rho\left(p_{r}^{k}, p_{r+1}^{k}\right)<1 / k$.

Some subsequence of $R_{1}, R_{2}, \cdots$ converges to a set $L$. But $L$ is connected, contains $p+q$, and belongs to both $M_{1}$ and $M_{2}$. Since $p$ and $q$ are arbitrary points of $M_{1} \cdot M_{2}$, we have that $M_{1} \cdot M_{2}$ is connected and $M$ is unicoherent.

Theorem 2. Suppose $G$ is a snake-like arc of continua $\left\{g_{x} \mid 0 \leqq x \leqq 1\right\}$ and $0<a<b<c<d<1$. There is a positive number $\epsilon$ such that if $D$ is any $\epsilon$-chain whatever covering $G$, any link of $D$ intersecting $G(b, c)$ is between any link of $D$ intersecting $G(0, a)$ and any link intersecting $G(d, 1)$.

Proof. The required number $\epsilon$ is any positive number less than each of $\rho(G(0, a), G(b, c)), \rho(G(b, c), G(d, 1))$, and $\rho(G(0, b), G(c, 1)) / 2$.

Let $D=d_{1}, d_{2}, \cdots, d_{n}$ be an $\epsilon$-chain covering $G$ and $d_{i}, d_{j}, d_{k}$ be elements of $D$ intersecting $G(0, a), G(b, c)$, and $G(d, 1)$ respectively where $i<k$. We show that $i<j<k$.

Since each of $G(0, b), G(c, 1)$ is connected, there are subchains $D^{\prime}, D^{\prime \prime}$ of $D$ containing $d_{i}$ and $d_{k}$ respectively and covering $G(0, b), G(c, 1)$ respectively such that each link of $D^{\prime}$ intersects $G(0, b)$ and each link of $D^{\prime \prime}$ intersects $G(c, 1)$. Since $\rho(G(0, b), G(c, 1))>2 \epsilon$, some link $d_{t}$ of $D$ lies between the links of $D^{\prime}$ and the links of $D^{\prime \prime}$. Hence $i<t<k$.

If $j<i<t, G(b, c)$ intersects $d_{i}$ because $G(b, c)$ is connected and intersects each of $d_{j}$ and $d_{t}$. This is impossible because $G(0, a)$ intersects $d_{i}$ and $\rho(G(0, a)$, $G(b, c))>\epsilon$. Similarly, it is impossible that $t<k<j$. Hence $i<j<k$.

Theorem 3. Suppose $G$ is a snake-like arc of continua $\left\{g_{x} \mid 0 \leqq x \leqq 1\right\}$. Then for each positive number $\epsilon$ there is an $\epsilon$-chain $D$ covering $G$ such that the first link of $D$ intersects $g_{0}$ and the last link intersects $g_{1}$.

Proof. Suppose $\delta$ is a positive number less than each of $\epsilon / 2$ and $\rho\left(g_{0}, g_{1}\right) / 2$. 
Since $G$ is snake-like, there is a $\delta$-chain $E=e_{1}, e_{2}, \cdots, e_{n}$ covering $G$. Suppose $e_{i}, e_{j}$ are the first and last members of this chain intersecting $g_{0}$ while $e_{r}, e_{s}$ are the first and last members of the chain intersecting $g_{1}$. Then $E(i, j)$ and $E(r, s)$ are subchains of $E$ properly covering $g_{0}$ and $g_{1}$ respectively and no link of $E(i, j)$ intersects any link of $E(r, s)$. For convenience we suppose that $i<j<r<s$.

Let $a, b$ be numbers such that $0<a<b<1, E(i, j)$ covers $G(0, a)$, and $E(r, s)$ covers $G(b, 1)$. An application of Theorem 2 gives a positive number $\gamma$ such that if $F$ is a $\gamma$-chain covering $G$, then any link of $F$ intersecting $G(a, b)$ lies between any link of $F$ intersecting $G(0, a / 2)$ and any intersecting $G((b+1) / 2,1)$. We put the further restriction on $\gamma$ that if $F$ is a $\gamma$-chain irreducibly covering $G$, then $F$ refines $E$, any link of $F$ intersecting $G(0, a)$ lies in a link of $E(i, j)$, and any link of $F$ intersecting $G(b, 1)$ lies in a link of $E(r, s)$.

Let $F=f_{1}, f_{2}, \cdots, f_{m}$ be a $\gamma$-chain irreducibly covering $G$ and $f_{t}, f_{u}$ be links of $F$ intersecting $g_{0}$ and $g_{1}$ respectively such that no link of $F$ between $f_{t}$ and $f_{u}$ intersects $g_{0}+g_{1}$. For convenience we suppose that $t<u$. Let $F(v, w)$ be the subchain of $F(t, u)$ which is maximal with respect to the property that $f_{v}$ lies in an end link of $E(i, j)$ and $f_{w}$ lies in an end link of $E(r, s)$. Then $E(i, j)$ covers each link of $F(1, v)$ and $E(r, s)$ covers each link of $F(w, m)$. For convenience we suppose that $f_{v}$ lies in $e_{j}$ and $f_{w}$ lies in $e_{r}$.

Use $A$ to denote the sum of the elements of $F(1, v)$ and $B$ to denote the sum of the elements of $F(v, m)$. Then the elements of $D$ are $A \cdot e_{i}, A \cdot e_{i+1}, \cdots$, $A \cdot e_{j}, f_{v+1}, f_{v+2}, \cdots, f_{w-1}, B \cdot e_{r}, B \cdot e_{r+1}, \cdots, B \cdot e_{s}$.

Theorem 4. Suppose $G$ is a snake-like arc of continua $\left\{g_{x} \mid 0 \leqq x \leqq 1\right\}$ and $p$ is a point of $G-\left(g_{0}+g_{1}\right)$. Then each neighborhood of $p$ which does not intersect $g_{0}+g_{1}$ separates $g_{0}$ from $g_{1}$ in $G$.

Proof. Suppose $U$ is a neighborhood of $p$ which does not intersect $g_{0}+g_{1}$ and $\epsilon$ is a positive number such that $\epsilon<\rho(p, G-U)$.

By Theorem 3 there is an $\epsilon$-chain $D=d_{1}, d_{2}, \cdots, d_{n}$ covering $G$ such that $d_{1}$ intersects $g_{0}$ and $d_{n}$ intersects $g_{1}$. Let $d_{i}$ be an element of $D$ that contains $p$. Then $G-U$ is the sum of the mutually exclusive closed sets $G \cdot\left(d_{1}+d_{2}+\cdots\right.$ $\left.+d_{i}\right)-U$ and $G \cdot\left(d_{i}+d_{i+1}+\cdots+d_{n}\right)-U$ while the first of these sets contains $g_{0}$ and the second contains $g_{1}$.

Theorem 5. Suppose $G$ is a snake-like arc of indecomposable continua $\left\{g_{x} \mid 0 \leqq x \leqq 1\right\}$ and $E$ is a chain covering $G$ such that $E$ properly covers each $g_{x}$. Then if $U$ is an open set such that $U \cdot G \neq 0, U \cdot\left(g_{0}+g_{1}\right)=0$, and $\bar{U}$ lies in a link of $E$, then $G-U$ may be expressed as the sum of two mutually separated sets $A, B$ such that $g_{0} \subset A, g_{1} \subset B$, and for each $x(0 \leqq x \leqq 1)$, either $A \cdot g_{x}$ intersects each link of $E$ or $B \cdot g_{x}$ intersects each link of $E$.

Proof. Let $D_{1}, D_{2}, \cdots$ be a decreasing sequence of chains covering $G$ such that the first link of each $D_{i}$ intersects $g_{0}$ and the last link intersects $g_{1}$. 
Theorem 3 shows that there are such chains.

Let $d_{1}$ be a link of some $D_{i}$ such that $d_{1} \subset U$ and diameter $d_{1}$ is less than 1 . Then Theorem 4 implies that $G-d_{1}$ is the sum of two mutually separated sets $A_{1}, B_{1}$ containing $g_{0}, g_{1}$ respectively. Using the assumption that the theorem is false, we find that there are numbers $a_{1}, b_{1}$ such that $0<a_{1}<b_{1}<1$ and for each $x\left(a_{1} \leqq x \leqq b_{1}\right)$, neither $A_{1} \cdot g_{x}$ nor $B_{1} \cdot g_{x}$ intersects each link of $E$.

Let $d_{2}$ be a link of some $D_{i}$ such that $d_{2} \subset d_{1}, d_{2} \cdot\left(G\left(0, a_{1}\right)+G\left(b_{1}, 1\right)\right)=0$, and diameter $d_{2}$ is less than $1 / 2$. Then $G-d_{2}$ is the sum of two mutually separated sets $A_{2}, B_{2}$ containing $G\left(0, a_{1}\right)$ and $G\left(b_{1}, 1\right)$ respectively. If the theorem is false, there are numbers $a_{2}, b_{2}$ such that $a_{1}<a_{2}<b_{2}<b_{1}$ and for each $g_{x}$ $\left(a_{2} \leqq x \leqq b_{2}\right)$ neither $A_{2} \cdot g_{x}$ nor $B_{2} \cdot g_{x}$ intersects each link of $E$.

We continue this procedure to get a sequence of open sets $d_{1}, d_{2}, d_{3}, \cdots$, a sequence of numbers $a_{1}, a_{2}, a_{3}, \cdots$ and a sequence of numbers $b_{1}, b_{2}, b_{3}, \ldots$ such that $d_{j+1} \subset d_{j}, d_{j}$ is of diameter less than $1 / j$ and is a link of some $D_{i}$, $a_{j}<a_{j+1}<b_{j+1}<b_{j}$, and $G-d_{j}$ is the sum of two mutually separated sets $A_{j}$, $B_{j}$ containing $G\left(0, a_{j-1}\right), G\left(b_{j-1}, 1\right)$ respectively.

Let $c$ be a number such that $0<a_{1}<a_{2}<\cdots<c<\cdots<b_{2}<b_{1}<1$. We show that the assumption that the theorem is false leads to the contradiction that $g_{c}$ is decomposable. For some increasing sequence of integers $n(1)$, $n(2), \cdots, A_{n(1)} \cdot g_{c}, A_{n(2)} \cdot g_{c}, \cdots$ converges to a set $A_{c}$ and $B_{n(1)} \cdot g_{c}, B_{n(2)}$ $\cdot g_{c}, \cdots$ converges to a set $B_{c}$. Now $A_{c}$ is a continuum because $A_{i} \cdot g_{c}$ is not the sum of two sets whose distance apart is more than $1 / i$. It is a proper subcontinuum of $g_{c}$ since no $A_{i} \cdot g_{c}$ intersects each link of $E$. Also, $B_{c}$ is a proper subcontinuum of $g_{c}$. But $g_{c}$ is decomposable because it is the sum of the two proper subcontinua $A_{c}, B_{c}$.

4. Chain maps. In this section we give some theorems about chain maps. The first of these might be labeled a fixed point theorem for chain maps.

Theorem 6. Suppose $D=d_{1}, d_{2}, \cdots, d_{n}$ and $E=e_{1}, e_{2}, \cdots, e_{m}$ are chains and $H_{1}, H_{2}$ are two chain maps of $D$ into $E$ such that $H_{i}(i=1,2)$ takes a link of $D$ into $e_{m-1}$. Then for some link $d_{i}$ of $D$, the link $H_{1}\left(d_{i}\right)$ of $E$ is adjacent to the link $H_{2}\left(d_{i}\right)$.

Proof. Suppose $H_{1}\left(d_{1}\right)$ precedes $H_{2}\left(d_{1}\right)$ in $e_{1}, e_{2}, \cdots, e_{m}$. If each link $H_{1}\left(d_{j}\right)$ of $E$ precedes the corresponding link $H_{2}\left(d_{j}\right)$, let $d_{i}$ be a link of $D$ such that $H_{1}\left(d_{i}\right)=e_{m-1}$. Then $H_{1}\left(d_{i}\right)$ is adjacent to $H_{2}\left(d_{i}\right)$.

If for some link $d_{j}$ of $D, H_{1}\left(d_{j}\right)$ does not precede $H_{2}\left(d_{j}\right)$, let $d_{i}$ be the first such link of $D$. Then $H_{1}\left(d_{i}\right)$ is adjacent to $H_{2}\left(d_{i}\right)$.

We note that if one chain covers a pseudo-arc, then another chain covering it can be inscribed in the first in a prescribed way.

Theorem 7. Suppose $D=d_{1}, d_{2}, \cdots, d_{n}$ is a chain properly covering a pseudo-arc $P$ and $H$ is a chain map of a chain $X=x_{1}, x_{2}, \cdots, x_{m}$ onto $D$. Then there is a chain $E=e_{1}, e_{2}, \cdots, e_{m}$ properly covering $P$ such that $e_{i} \subset d_{j}$ if $H\left(x_{i}\right)=d_{j}$. 
In fact, if $A, B$ are closed sets in $P \cdot d_{1}, P \cdot d_{n}$ respectively and $H\left(x_{r}\right)=d_{1}$, $H\left(x_{s}\right)=d_{n}$, the chain $E$ may be selected so that $A \subset e_{r}, B \subset e_{s}$.

Proof. The proof would be slightly easier if $A \subset d_{1}-\bar{d}_{2}, B \subset d_{n}-\bar{d}_{n-1}, r=1$, $s=m, x_{r}$ is the only element of $X$ that $H$ takes into $d_{1}$, and $x_{s}$ is the only element of $X$ that $H$ takes into $d_{n}$. We now prove the theorem in this special case.

Let $p_{1}, p_{2}$ be points of different components of $P$ in $d_{1}-d_{2}, d_{n}-d_{n-1}$ respectively and $D_{1}, D_{2}, \cdots$ be a sequence of chains from $p_{1}$ to $p_{2}$ such that each $D_{i}$ covers $P, D_{i}$ is of mesh less than $1 / i, D_{i+1}$ is crooked in $D_{i}$, and $D=D_{1}$. Then it follows from Theorem 6 of [3] that there is a chain $E=e_{1}$, $e_{2}, \cdots, e_{m}$ from $p_{1}$ to $p_{2}$ such that $E$ covers $P, e_{i} \subset d_{j}$ if $H\left(x_{i}\right)=d_{j}$, and for some integer $r$, each link of $E$ is the sum of links of $D_{r}$.

Now that we have shown the theorem is true in the special case we alter $D, X, H$ and obtain $D^{\prime}, X^{\prime}, H^{\prime}$ so that the special case applies. Then we adjust the $E^{\prime}$ obtained to get the required $E$. We suppose with no loss of generality that $r<s$.

The chain $D^{\prime}$ is obtained as follows. Let $U_{0}, U_{1}, U_{2}$ be open subsets of $d_{1}, d_{1} \cdot d_{2}, d_{2}$ respectively such that $P \cdot\left(d_{1}+d_{2}\right) \subset U_{0}+U_{1}+U_{2}, P \cdot\left(d_{1}-d_{2}\right)$ $+A \subset U_{0}, P \cdot\left(d_{2}-d_{1}\right) \subset U_{2}, U_{0} \cdot \bar{U}_{2}=0$, and $A \cdot \bar{U}_{1}=0$. Also, let $U_{n-1}, U_{n}, U_{n+1}$ be open subsets of $d_{n-1}, d_{n-1} \cdot d_{n}, d_{n}$ respectively such that $P \cdot\left(d_{n-1}+d_{n}\right) \subset U_{n-1}$ $+U_{n}+U_{n+1}, P \cdot\left(d_{n-1}-d_{n}\right) \subset U_{n-1}, P \cdot\left(d_{n}-d_{n-1}\right)+B \subset U_{n+1}, \bar{U}_{n-1} \cdot \bar{U}_{n+1}=0$, and $\bar{U}_{n} \cdot B=0$. Then $D^{\prime}=U_{0}, U_{1}, U_{2}, d_{3}, \cdots, d_{n-2}, U_{n-1}, U_{n}, U_{n+1}$.

The chain $X^{\prime}=y^{\prime}, y_{r}, y_{r-1}, \cdots, y_{2}, x_{1}, x_{2}, \cdots, x_{m}, z_{m-1}, \cdots, z_{s}, z^{\prime}$ is obtained from $X$ by adding $r$ elements on at the front and $m+1-s$ on at the end. Then $H^{\prime}$ is the chain map of $X^{\prime}$ onto $D^{\prime}$ so that $H^{\prime}\left(y^{\prime}\right)=U_{0}, H^{\prime}\left(z^{\prime}\right)$ $=U_{n+1}, H^{\prime}\left(x_{i}\right)=H^{\prime}\left(y_{i}\right)=H^{\prime}\left(z_{i}\right)=U_{j}$ (or $\left.d_{j}\right)$ if $H\left(x_{i}\right)=d_{j}$.

Since the special case applies to $D^{\prime}, X^{\prime}$, and $H^{\prime}$, there is a chain $E^{\prime}=f^{\prime}$, $f_{r}, f_{r-1}, \cdots, f_{2}, e_{1}^{\prime}, e_{2}^{\prime}, \cdots, e_{m}^{\prime}, g_{m-1}, \cdots, g_{s}, g^{\prime}$ such that $A \subset f^{\prime}, B \subset g^{\prime}$, and a link $e^{\prime}$ of $E^{\prime}$ lies in a link $d$ of $D^{\prime}$ provided $d$ is the image under $H^{\prime}$ of the link of $X^{\prime}$ corresponding to $e^{\prime}$.

The chain $E=e_{1}, e_{2}, \cdots, e_{m}$ satisfying the conclusions of the theorem is obtained by adding together certain elements of $E^{\prime}$. The link $e_{r}$ is the sum of $f^{\prime}, f_{r}, e_{r}^{\prime}$ while $e_{s}$ is the sum of $g^{\prime}, g_{s}, e_{s}^{\prime}$. In general $e_{i}$ is the sum of $e_{i}^{\prime}, f_{i}, g_{i}$ (if there are such $f$ 's and $g$ 's).

The following theorem concerning the existence of chains that cover a snake-like arc of pseudo-arcs in a prescribed fashion will be of use in showing that certain such continua are homeomorphic.

Theorem 8. Suppose $P$ is a snake-like arc of pseudo-arcs $\left\{p_{x} \mid 0 \leqq x \leqq 1\right\}$, $D=d_{1}, d_{2}, \cdots, d_{n}$ is a chain covering $P$ such that each link of $D$ intersects each $p_{x}$, and $H$ is a chain map of a chain $Y=y_{1}, y_{2}, \cdots, y_{m}$ onto $D$. Then there is a chain $E=e_{1}, e_{2}, \cdots, e_{m}$ covering $P$ such that each $p_{x}$ intersects each link of $E$ and $e_{i} \subset d_{j}$ if $H\left(y_{i}\right)=d_{j}$. 
Proof. First we consider the case where $H\left(y_{1}\right)=d_{1}, H\left(y_{m}\right)=d_{n}$. Let $U_{A}, U_{B}$ be open sets with closures in $d_{1}, d_{2}$ respectively such that $U_{A}, U_{B}$ intersect each element of $\left\{p_{x}\right\}$.

It follows from Theorem 7 that for each $a(0 \leqq a \leqq 1)$ there is a chain $E(a)=e(a)_{1}, e(a)_{2}, \cdots, e(a)_{m}$ covering $p_{a}$ such that $\bar{U}_{A} \cdot p_{a} \subset e(a)_{1}, \bar{U}_{B} \cdot p_{a}$ $\subset e(a)_{m}, e(a)_{i} \subset d_{j}$ if $H\left(y_{i}\right)=d_{j}$.

The upper semicontinuity of the collection $\left\{p_{x}\right\}$ implies that $a$ lies in a connected open subset $I_{a}$ of $(0 \leqq x \leqq 1)$ such that if $x \in I_{a}$, then $E(a)$ covers $p_{x}, \bar{U}_{A} \cdot p_{x} \subset e(a)_{1}$, and $\bar{U}_{B} \cdot p_{x} \subset e(a)_{m}$. A finite collection $I_{a_{1}}, I_{a_{2}}, \cdots, I_{a_{t}}$ of such open connected subsets cover $(0 \leqq x \leqq 1)$. Let $E\left(a_{1}\right), E\left(a_{2}\right), \cdots, E\left(a_{t}\right)$ be the corresponding $E(a)$ 's.

We now show how to form $E$ from $E\left(a_{1}\right), E\left(a_{2}\right), \cdots E\left(a_{t}\right)$. It follows from Theorem 4 that $P$ is covered by open sets $U_{1}, U_{2}, \cdots, U_{t}$ such that $U_{i} \cdot U_{j}=U_{A}+U_{B}$ if $i \neq j$ and if $p_{x} \cdot\left(U_{i}-\left(U_{A}+U_{B}\right)\right) \neq 0$, then $x \in I_{a_{i}}$. Then the chain $E$ whose $k$ th link $e_{k}=e\left(a_{1}\right)_{k} \cdot U_{1}+e\left(a_{2}\right)_{k} \cdot U_{2}+\cdots+e\left(a_{t}\right)_{k} \cdot U_{t}$ is the required chain.

The purpose of the restriction that $H\left(y_{1}\right)=d_{1}, H\left(y_{m}\right)=d_{n}$ was to make it apparent that each $p_{x}$ intersected each link of $E$. To prove the theorem in the more general case, suppose $H\left(y_{r}\right)=d_{1}, H\left(y_{s}\right)=d_{n}$. Then alter $Y$ as was done in the proof of Theorem 7 by adding elements onto the first of it and elements onto the last of it so that the resulting chain $Y^{\prime}$ has ends in $d_{1}, d_{n}$ respectively and such that the added elements of $Y^{\prime}$ can be combined with the original links to get a chain resembling $Y$. Then end chains of the resulting chain $E^{\prime}$ may be added onto the center of $E^{\prime}$ as was done in the proof of Theorem 7 so as to get a chain $E$ satisfying the conditions of the theorem.

In stating the following corollary to Theorem 8 , the same symbols are used as appear at the place in the proof of Theorem 10 where it is applied.

Corollary to Theorem 8. Suppose $Q(i / k,(i+1) / k)$ is a snake-like arc of pseudo-arcs $\left\{q_{x} / i / k \leqq x \leqq(i+1) / k\right\}, E$ is a chain covering $Q(i / k,(i+1) / k)$ such that each $q_{x}$ intersects each link of $E$, and $H_{1}$ is a chain map of a chain $D(r, s)$ onto $E$. Then there is a positive number $\epsilon$ such that if $F(t, u)$ is an $\epsilon-$ chain irreducibly covering $Q(i / k,(i+1) / k)$, then there is a chain map $R_{(2 i+1) / 2 k}$ of $F(t, u)$ onto $D(r, s)$ such that for each link $f_{i}$ of $F(t, u), f_{i} \subset H_{1} R_{(2 i+1) / 2 k}\left(f_{i}\right)$, and if $f_{v}, f_{v+1}, \cdots, f_{w}$ is a subchain of $F(t, u)$ irreducibly covering some $q_{x}$, then each link of $D(r, s)$ is the image of three consecutive links of $f_{v}, f_{v+1}, \cdots, f_{w}$.

In obtaining this result from Theorem 8 , we let the $P,\left\{p_{x} \mid 0 \leqq x \leqq 1\right\}$, and $D$ of the theorem be the $Q(i / k,(i+1) / k),\left\{q_{x} \mid i / k \leqq x \leqq(i+1) / k\right\}$, and $E$ of the corollary, $Y$ be a chain with four times as many links as $D(r, s)$, and $H$ be a chain map that takes links of $Y$ numbered $4 i+1,4 i+2,4 i+3,4 i+4$ into the image under $H_{1}$ of the $i$ th link of $D(r, s)$. Then $\epsilon$ is a number so small that any $\epsilon$-chain properly covering $Q(i / k,(i+1) / k)$ is a refinement of the chain $E$ promised by Theorem 8 . 
5. A homeomorphism between pseudo-arcs. The following theorem shows that if the same $\epsilon$-chain properly covers two pseudo-arcs, there is a homeomorphism of one onto the other that moves no point by more than $\epsilon$. It also shows how a chain map can be approximated with a homeomorphism.

Theorem 9. Suppose $D, E$ are chains properly covering pseudo-arcs $P, Q$ and $H$ is a chain map of $D$ onto $E$ such that each link of $E$ is the image of a link of $D$ which contains a point of $P$ not on the closure of any other link of $D$. Then there is a homeomorphism $h$ of $P$ onto $Q$ such that for each link $d_{i}$ of $D, h\left(P \cdot d_{i}\right)$ $\subset H\left(d_{i}\right)$.

The rather awkward condition that each link of $E$ is the image of a link of $D$ that covers a point of $P$ not covered by the closure of any other link of $D$ is necessary because it may be that $D=d_{1}, d_{2}, \cdots, d_{n}$ and $E=e_{1}, e_{2}, \cdots, e_{n}$ have the same number of links, $H\left(d_{i}\right)=e_{i}, P \subset \bar{d}_{2}+\bar{d}_{3}+\cdots+\bar{d}_{n-1}$, and $Q \nsubseteq \bar{e}_{2}+\bar{e}_{3}+\cdots+\bar{e}_{n-1}$. The less complicated but more stringent condition that each link of $E$ is the image of an interior link of $D$ might be substituted.

We use two simplifications in this proof so as to make the remaining part of the proof essentially like the proof of Theorem 12 of [3].

First SIMPLIfication. We now show that there is no loss of generality in supposing that $D$ and $E=e_{1}, e_{2}, \cdots, e_{n}$ have the same number of links, $H$ takes the $i$ th link of $D$ into the $i$ th link of $E$, and each end link of $D$ contains a point not on the closure of any other link of $D$.

Let $D^{\prime \prime}=d_{1}^{\prime \prime}, d_{2}^{\prime \prime}, \cdots, d_{n}^{\prime \prime}$ be the chain such that $d_{i}^{\prime \prime}$ is the sum of the links of $D$ that go in to the $i$ th link of $E$ under $H$. Then $D^{\prime \prime}$ is a chain covering $P$ such that each end link of $D^{\prime \prime}$ contains a point not contained on the closure of any other link of $D^{\prime \prime}$. Also, if $h$ is a homeomorphism of $P$ onto $Q$ such that $h\left(P \cdot d_{i}^{\prime \prime}\right) \subset e_{i}$, then $h$ is the required homeomorphism. Hence we suppose with no loss of generality that $D^{\prime \prime}=d_{1}^{\prime \prime}, d_{2}^{\prime \prime}, \cdots, d_{n}^{\prime \prime}=d_{1}, d_{2}, \cdots, d_{n}=D$, $H\left(d_{i}\right)=e_{i}, P \cdot\left(d_{1}-\bar{d}_{2}\right)$ contains a point $p_{1}$, and $P \cdot\left(d_{n}-\bar{d}_{n-1}\right)$ contains a point $p_{2}$ such that $p_{1}, p_{2}$ belong to different composants of $P$.

SeCOND Simplification. We replace chains $D=d_{1}, d_{2}, \cdots, d_{n}$ and $E=e_{1}, e_{2}, \cdots, e_{n}$ left after the first simplification with chains $D^{\prime}=d_{1}^{\prime}$, $d_{2}^{\prime}, \cdots, d_{4 n-3}^{\prime}$ and $E^{\prime}=e_{1}^{\prime}, e_{2}^{\prime}, \cdots, e_{4 n-3}^{\prime}$ covering $P$ and $Q$ respectively such that $p_{1} \in\left(d_{1}^{\prime}-\bar{d}_{2}^{\prime}\right), p_{2} \in\left(d_{4 n-3}^{\prime}-\bar{d}_{4 n-4}^{\prime}\right)$, and if $h$ is any homeomorphism of $P$ onto $Q$ such that $h\left(P \cdot d_{i}^{\prime}\right) \subset \bar{e}_{i-1}^{\prime}+\bar{e}_{i}^{\prime}+\bar{e}_{i+1}^{\prime}$, then $h$ is the required homeomorphism.

First we suppose that $q_{1}, q_{2}$ are points of different composants of $Q$ in $e_{1}-e_{2}$ and $e_{n}-e_{n-1}$ respectively. If there are not already such points, points may be deleted from $e_{2}$ and $e_{n-1}$ to make the condition satisfied.

Suppose that $\epsilon$ is a positive number so small that each subset of $Q$ of diameter less than $5 \epsilon$ lies in one link of $E$. Let $F$ be an $\epsilon$-chain properly covering $Q$. Then $e_{1}^{\prime}$ is the sum of all links of $F$ whose closures intersect $e_{1}-e_{2}$, $e_{5}^{\prime}$ is the sum of all links of $F$ whose closures intersect $e_{2}-\left(e_{1}+e_{3}\right), e_{9}^{\prime}$ is the 
sum of all links of $F$ whose closures intersect $e_{3}-\left(e_{2}+e_{4}\right), \cdots$, and $e_{4 n-3}^{\prime}$ is the sum of all links of $F$ whose closures intersect $e_{n}-e_{n-1}$. The links of $F$ with closures in $e_{1} \cdot e_{2}$ are combined to form $e_{2}^{\prime}, e_{3}^{\prime}, e_{4}^{\prime}$; the links of $F$ with closures in $e_{2} \cdot e_{3}$ are combined to form $e_{6}^{\prime}, e_{7}^{\prime}, e_{8}^{\prime}, \cdots$, and the links of $G$ with closures in $e_{n-1} \cdot e_{n}$ are combined to form $e_{4 n-6}^{\prime}, e_{4 n-5}^{\prime}, e_{4 n-4}^{\prime}$. (See Figure 1.)
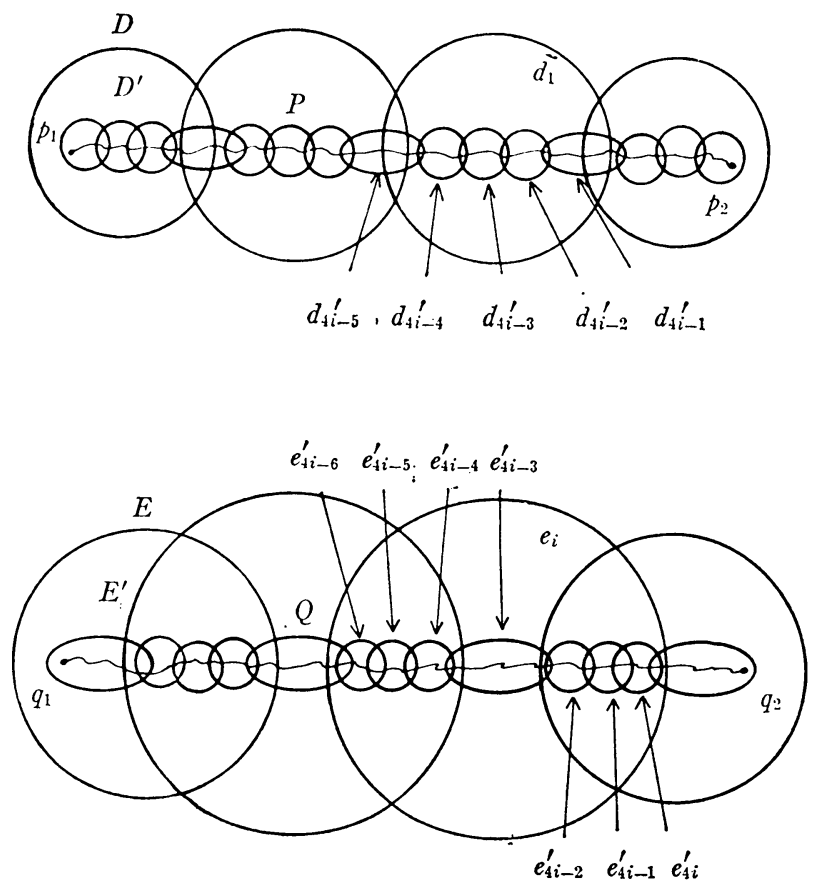

FIG. 1

Let $\delta$ be a positive number so small that the distance between any pair of nonadjacent links of $D=d_{1}, d_{2}, \cdots, d_{n}$ is more than $5 \delta$ and $\rho\left(p_{1}+p_{2}, d_{2}+d_{n-1}\right)$ $>3 \delta$. Let $G$ be a $\delta$-chain properly covering $P$ and refining $D$. Then $d_{3}^{\prime}$ is the sum of the links of $G$ that intersect $d_{1} \cdot d_{2}, d_{7}^{\prime}$ is the sum of the links of $G$ that intersect $d_{2} \cdot d_{3}, \cdots$, and $d_{4 n-5}^{\prime}$ is the sum of the links of $G$ that intersect $d_{n-1} \cdot d_{n}$. Also, $d_{1}^{\prime}$ is the sum of the links of $G$ whose closures contain $p_{1} ; d_{2}^{\prime}$ is the sum of the other links of $G$ in $e_{1}-e_{2} ; d_{4}^{\prime}, d_{5}^{\prime}, d_{6}^{\prime}$ are formed by combining links of $G$ in $e_{2}-\left(e_{1}+e_{3}\right), \cdots, d_{4 n-3}^{\prime}$ is the sum of the links of $G$ whose closures contain $p_{2} ; d_{4 n-4}^{\prime}$ is the sum of the other links of $G$ in $e_{n}-e_{n-1}$. See Figure 1.

We now show that if $h$ is a homeomorphism of $P$ onto $Q$ such that $h\left(P \cdot d_{i}^{\prime}\right) \subset \bar{e}_{i-1}^{\prime}+\bar{e}_{i}^{\prime}+\bar{e}_{i+1}^{\prime}$, then $h$ is the required homeomorphism because $h\left(P \cdot d_{i}\right) \subset e_{i}$. The reason is that $h\left(P \cdot d_{i}\right) \subset h\left(P \cdot\left(d_{4 i-5}^{\prime}+d_{4 i-4}^{\prime}+d_{4 i-3}^{\prime}+d_{4 i-2}^{\prime}\right.\right.$ $\left.\left.+d_{4 i-1}^{\prime}\right)\right) \subset \bar{e}_{4 i-6}^{\prime}+\bar{e}_{4 i-5}^{\prime}+\bar{e}_{4 i-4}^{\prime}+\bar{e}_{4 i-3}^{\prime}+\bar{e}_{4 i-2}^{\prime}+\bar{e}_{4 i-1}^{\prime}+\bar{e}_{4 i}^{\prime} \bar{e}_{i}$.

Completion of proof. It follows from Theorem 13 of [3] and the definition 
of a pseudo-arc that there is a sequence of chains $F_{1}, F_{2}, \ldots$ from $p_{1}$ to $p_{2}$ such that $F_{i}$ covers $P, F_{i+1}$ is crooked in $F_{i}$, and $F_{i}$ is of mesh less than $1 / i$. Also, there is a sequence of chains $G_{1}, G_{2}, \cdots$ from $q_{1}$ to $q_{2}$ such that $G_{i}$ covers $Q, G_{i+1}$ is crooked in $G_{i}$, and $G_{i}$ is of mesh less than $1 / i$.

In order to define the homeomorphism $h$ we shall obtain a sequence of chains $D_{1}, D_{2}, \ldots$ from $p_{1}$ to $p_{2}$ and a sequence of chains $E_{1}, E_{2}, \cdots$ from $q_{1}$ to $q_{2}$ such that (1) $D_{i}$ covers $P$ and $E_{i}$ covers $Q$; (2) $D_{i}, E_{i}(i>2)$ are of mesh less than $1 / i$; and (3) $D_{i}=d_{1}^{i}, d_{2}^{i}, \cdots, d_{n_{i}}^{i}$ and $E_{i}=e_{1}^{i}, e_{2}^{i}, \cdots, e_{n_{i}}^{i}$ have the same number of links and there is a chain map $H_{i}$ of $E_{i+1}$ onto $E_{i}$ and $D_{i+1}$

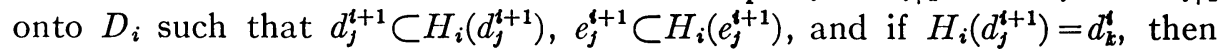
$H_{i}\left(e_{j}^{i+1}\right)=e_{k}^{t}$.

We set $D_{1}=D^{\prime}$ and $E_{1}=E^{\prime}$ where $D^{\prime}$ and $E^{\prime}$ are the chains obtained in the second simplification.

Let $E_{2}$ be any $G_{i}$ of mesh less than $1 / 3$ that refines $E_{i}$ and $H_{2}$ be any chain map of $E_{2}$ onto $E_{1}$ such that $e_{j}^{2} \subset H_{1}\left(e_{j}^{2}\right)$ for each $j$. Then by Theorem 6 of [3] we find that there is a chain $D_{2}$ from $p_{1}$ to $p_{2}$ covering $P$ such that for some integer $r$, each link of $D_{2}$ is the sum of links of $F_{r}, D_{2}$ has the same number of links as $E_{2}$, and $d_{j}^{2} \subset d_{\mathfrak{k}}^{1}=H_{1}\left(d_{j}^{2}\right)$ if $H_{1}\left(e_{j}^{2}\right)=d_{\mathfrak{k}}^{1}$.

Let $D_{3}$ be any $F_{i}$ of mesh less than $1 / 4$ that refines $D_{2}$ and $H_{2}$ be any chain map of $D_{3}$ onto $D_{2}$ such that $d_{j}^{3} \subset H_{2}\left(d_{j}^{3}\right)$ for each $j$. Again it follows from Theorem 6 of [3] that there is a chain $E_{3}$ from $q_{1}$ to $q_{2}$ covering $Q$ such that for some integer $r$, each link of $E_{3}$ is the sum of links of $G_{r}, E_{3}$ has the same number of links as $D_{3}$, and $e_{j}^{3} \subset e_{\mathbf{k}}^{2}=H_{2}\left(e_{j}^{3}\right)$ if $H_{2}\left(d_{j}^{3}\right)=d_{\mathbf{k}}^{2}$.

The sequences $D_{1}, D_{2}, \cdots$ and $E_{1}, E_{2}, \cdots$ are obtained by a repetition of this procedure.

Let $d(p, i)$ denote the sum of the elements of $D_{i}$ containing the point $p$ of $P$ and $e(p, i)$ denote the sum of the corresponding elements of $E_{i}$. Then $d(p, i+1) \subset d(p, i)$. Also, $e(p, i+1) \subset e(p, i)$ since if $e_{j}^{i+1}$ lies in $e(p, i+1), d_{j}^{i+1}$ contains $p, H_{i}\left(d_{j}^{t+1}\right)=d_{\boldsymbol{k}}^{t}$ contains $p$ and lies in $d(p, i)$, and $e_{\mathbf{k}}^{i}$ lies in $e(p, i)$ and contains $e_{j}^{i+1}$. The homeomorphism $h$ is defined so that $h(p)$ is the intersection of the closures of the decreasing sequence of open sets $e(p, 1), e(p, 2), \cdots$. That $h$ is a homeomorphism of $P$ onto $Q$ follows from an argument similar to that contained at the end of the proof of Theorem 10 of this paper or in Theorem 11 of [3].

Finally we show that $h\left(P \cdot d_{i}^{1}\right) \subset \bar{e}_{i-1}^{1}+\bar{e}_{i}^{1}+\bar{e}_{i+1}^{1}$. If $p$ is a point of $d_{i}^{1}, d(p, 1)$ $\subset d_{i-1}^{1}+d_{i}^{1}+d_{i+1}^{1}$ and $h(p) \in \bar{e}(p, 1) \subset \bar{e}_{i-1}^{1}+\bar{e}_{i}^{1}+\bar{e}_{i+1}^{1}$. Hence $h\left(P \cdot d_{i}^{1}\right) \subset \bar{e}_{i-1}^{1}+\bar{e}_{i}^{1}$ $+\bar{e}_{t+1}^{1}$.

6. Homeomorphisms between arcs of pseudo-arcs. The theorem of this section shows that there is a strong type of topological equivalence between any two continuous snake-like arcs of pseudo-arcs. It is a key theorem to the showing that each circle of pseudo-arcs is homogeneous.

Theorem 10. Suppose $P, Q$ are continuous snake-like arcs of pseudo-arcs $\left\{p_{x}\right\},\left\{q_{x}\right\}(0 \leqq x \leqq 1)$. Then each homeomorphism $h$ that takes the sum of the 
ends of $P$ onto the sum of the ends of $Q$ may be extended to a homeomorphism taking $P$ onto $Q$. In fact, if $h\left(p_{0}\right)=q_{0}$, the extended homeomorphism $h$ may be chosen so that $h\left(p_{x}\right)=q_{x}$.

Proof. Here is an outline of our plan. We shall get a sequence of chains $D_{1}, D_{2}, \cdots$ covering $P$ and a sequence of chains $E_{1}, E_{2}, \cdots$ covering $Q$ and use these sequences to define our homeomorphism $h$. Also, we obtain sequences of chain maps $H_{1}, H_{2}, \cdots$ and $K_{1}, K_{2}, \cdots$ such that $H_{i}$ takes $D_{i}$ onto $E_{i}$ and $K_{i}$ takes $E_{i+1}$ onto $D_{i}$. This is illustrated as follows:

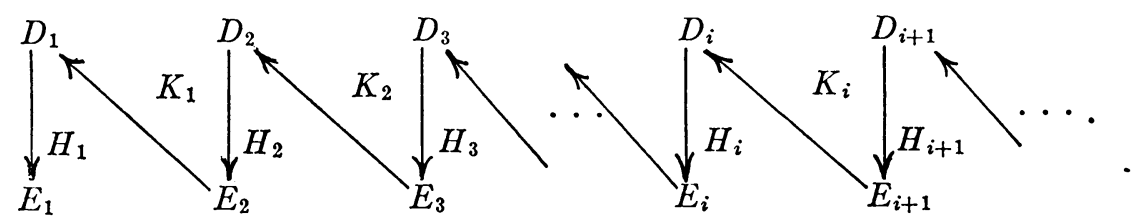

The chain map $H_{1}$ will be an approximation to the homeomorphism $h$ we are seeking in that for each point $p$ of $P$, there is an integer $i$ such that $p$ belongs to the $i$ th link $d_{i}^{1}$ of $D_{1}$ and $h(p)$ belongs to the link $H_{1}\left(d_{i}^{1}\right)$ of $E_{1}$. Also, $K_{1}$ will be an approximation to $h^{-1}$ in that for each point $q$ of $Q$ there is an integer $i$ such that $q$ is an element of the $i$ th link $e_{i}^{2}$ of $E_{2}$ and $h^{-1}(q)$ is in the link $K_{1}\left(e_{\imath}^{2}\right)$ of $D_{1}$. Furthermore, $K_{1}$ agrees with $H_{1}^{-1}$ in that each link of $E_{2}$ lies in its image under $H_{1} K_{1}$.

The $D$ 's, $E$ 's, $H$ 's, $K$ 's will be defined in the following order: $E_{1}, D_{1}, H_{1}$, $E_{2}, K_{1}, D_{2}, H_{2}, E_{3}, K_{3}, \cdots$. The meshes of $D_{i}$ and $E_{i}$ are less than $1 / 2^{i}$. In general, $e_{j}^{i+1}$ is contained in $H_{i} K_{i}\left(e_{j}^{i+1}\right)$ where $e_{j}^{i+1}$ is the $j$ th link of $E_{i+1}$ and $d_{j}^{i+1}$ is contained in $K_{i} H_{i+1}\left(d_{j}^{i+1}\right)$. (See Figure 2.)

The $D$ 's, $E$ 's, $H$ 's and $K$ 's will be chosen so that for each point $p$ of $p_{x}$, there is a decreasing sequence of links $d_{n_{1}}^{1}, d_{n_{2}}^{2}, \cdots$ containing $p\left(d_{n_{i}}^{i} \in D_{i}\right)$ such that $K_{i-1} H_{i}\left(d_{n_{i}}^{i}\right)=d_{n_{i-1}}^{i-1}$ and $H_{1}\left(d_{n_{1}}^{1}\right), H_{2}\left(d_{n_{2}}^{2}\right), \cdots$ is a sequence of links $\left(H_{i}\left(d_{n_{i}}^{i}\right) \in E_{i}\right)$ whose closures contain a point $q$ of $q_{x}$. The homeomorphism $h$ is chosen so that $h(p)=q$. In order to define the $D$ 's, $E$ 's, $H$ 's, and $K$ 's properly, we extend the homeomorphism $h$ to certain elements of $\left\{p_{x}\right\}$ as we go.

We shall use $P(a, b)$ to denote the sum of the elements of $\left\{p_{x} \mid a \leqq x \leqq b\right\}$ and $Q(a, b)$ to denote the sum of the elements of $\left\{q_{x} \mid a \leqq x \leqq b\right\}$.

Now for the details of the proof.

Step 1. In this step we define $E_{1}$ and extend the homeomorphism $h$ to some more elements of $\left\{p_{x}\right\}$. The step contains three parts.

(a) Consider a chain $E_{1}$ irreducibly covering $Q$ and of mesh less than $1 / 2$.

(b) It follows from the continuity of the collection $\left\{p_{x}\right\}$ and the bicompactness of an arc that there is a positive integer $n$ such that if $0 \leqq b-a$ $\leqq 1 / n$, then the subchain of $E_{1}$ that irreducibly covers $Q(a, b)$ properly covers each element of $\left\{q_{x} / a \leqq x \leqq b\right\}$.

(c) Extend the homeomorphism $h$ already defined on $p_{0}+p_{1}$ to $p_{0}+p_{1 / n}$ 


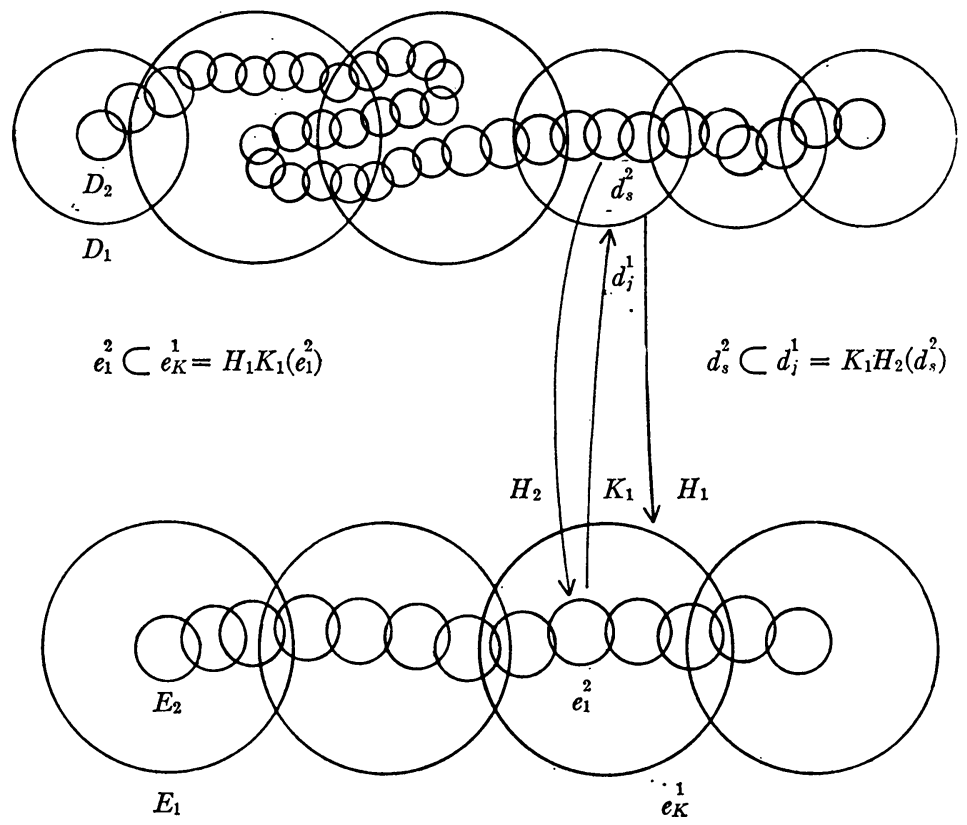

FIG. 2

$+p_{2 / n}+\cdots+p_{1}$. We do not need to exercise the care in making this extension that we will need to do in later steps. We merely impose the condition that $h$ takes $p_{i / n}$ homeomorphically onto $q_{i / n}$.

Step 2. In this step we define $D_{1}, H_{1}$, and extend the map $h$ still further by defining it on more elements of $\left\{p_{x}\right\}$. We wish the following conditions to be satisfied.

I. If link $d_{j}$ of $D_{1}$ intersects $p_{i / n}$, then $H_{1}\left(d_{j}\right)$ contains $h\left(d_{j} \cdot p_{i / n}\right)$.

II. For each $x, H_{1}$ takes any subchain of $D_{1}$ that properly covers $p_{x}$ onto a subchain of $E_{1}$ that properly covers $q_{x}$.

Parts (a)-(e) of Step 2 are used in getting $D_{1}$ and $H_{1}$. Parts (f)-(h) show that $H_{1}$ and $D_{1}$ satisfy the above Conditions I and II. In Part (i) we extend the homeomorphism to other elements of $\left\{p_{x}\right\}$.

(a). In Step 2a we get a preliminary approximation $F$ to the chain $D_{1}$. We let $F$ be an $\epsilon$-chain covering $P$ where $\epsilon$ is small enough to make the following statements true. (1) $\epsilon<1 / 2$. (2) No link of $F$ intersects both $p_{a}$ and $p_{b}$ if $1 / 2 n \leqq b-a$. (3) The image under $h$ of any subset of $p_{0}+p_{1 / n}+\cdots+p_{1}$ of diameter less than $5 \epsilon$ lies in an element of $E_{1}$. (4) Any subchain of $F$ covering an element of $\left\{p_{x}\right\}$ has at least six times as many links as $E_{1}$.

(b) In this part of Step 2 we get approximations $R_{0}, R_{1 / 2 n}, R_{1 / n}, R_{3 / 2 n}, \cdots$, $R_{1}$ to $H_{1}$.

First we describe $R_{i / n}(i=0,1, \cdots, n)$. Consider the subchain $f_{t}, f_{t+1}, \cdots, f_{u}$ of $F$ irreducibly covering $p_{i / n}$ and the subchain $e_{r}, e_{r+1}, \cdots, e_{8}$ of $E_{1}$ ir- 
reducibly covering $q_{i / n}$. Then $R_{i / n}$ is a chain map of $f_{t}, f_{t+1}, \cdots, f_{u}$ onto $e_{r}, e_{r+1}, \cdots, e_{s}$ such that $h\left(f_{j} \cdot p_{i / n}\right) \subset R_{i / n}\left(f_{j}\right)$ and for each element $e_{k}$ of $e_{r}$, $e_{r+1}, \cdots, e_{s}$ there are three consecutive elements of $f_{t}, f_{t+1}, \cdots, f_{u}$ that go into $e_{k}$ under $R_{i / n}$. A precaution to take to insure that each $e_{k}$ is the image of three consecutive elements of $f_{t}, f_{t+1}, \cdots, f_{u}$ is to decide that $R_{i / n}\left(f_{j-2}\right)$ $=R_{i / n}\left(f_{j-1}\right)=R_{i / n}\left(f_{j}\right)=R_{i / n}\left(f_{j+1}\right)=R_{i / n}\left(f_{j+2}\right)=e_{k}$ if $e_{k}$ is the only element of $E_{1}$ such that $h\left(f_{j} \cdot p_{i / n}\right) \subset e_{k}$. Condition 3 of Step 2 a enables us to do this. Unless $f_{j}$ is the first or last element of $F$, such an $R_{i / n}$ sends more than three consecutive elements of $F$ into $e_{k}$.

Now we describe $R_{(2 i+1) / 2 n}(i=0,1, \cdots, n-1)$. Let $f_{t}, f_{t+1}, \cdots, f_{u}$ and $e_{r}, e_{r+1}, \cdots, e_{s}$ be the subchains of $F$ and $E_{1}$ that irreducibly cover $P(i / n$, $(i+1) / n)$ and $Q(i / n,(i+1) / n)$ respectively. It may be that the subscripts $t$ and $u$ mentioned here may differ considerably from the $t$ and $u$ mentioned in the last paragraph but the $r$ and $s$ used here do not differ by more than 1 from those used there. It follows from Step $1 \mathrm{~b}$ that $e_{r}, e_{r+1}, \cdots, e_{s}$ properly covers each element of $\left\{q_{x} / i / n \leqq x \leqq(i+1) / n\right\}$. Then $R_{(2 i+1) / 2 n}$ is any chain map whatever of $f_{t}, f_{t+1}, \cdots, f_{u}$ onto $e_{r}, e_{r+1}, \cdots, e_{s}$ so long as it is true that for each element $e_{k}$ of $e_{r}, e_{r+1}, \cdots, e_{s}$ and each subchain $f_{v}$, $f_{v+1}, \cdots, f_{w}$ of $f_{t}, f_{t+1}, \cdots, f_{u}$ irreducibly covering an element of $\left\{p_{x} \mid i / n\right.$ $\leqq x \leqq(i+1) / n\}$ there are three consecutive elements of $f_{v}, f_{v+1}, \cdots, f_{w}$ that go into $e_{k}$ under $R_{(2 i+1) / 2 n}$. We must be more careful in describing the analogue of $R_{(2 i+1) / 2 n}$ in Step 3 but we can accomplish the result here by letting $R_{(2 i+1) / 2 n}$ send the first three elements of $f_{t}, f_{t+1}, \cdots, f_{u}$ into $e_{r}$; the next three into $e_{r+1}, \cdots$, the next three into $e_{8}$, the next three into $e_{s-1}, \cdots$. Condition 4 of Step 2a assures us that $f_{v}, f_{v+1}, \cdots, f_{w}$ has enough elements that such a procedure will cause each element of $e_{r}, e_{r+1}, \cdots, e_{s}$ to be the image of some three consecutive members of $f_{v}, f_{v+1}, \cdots, f_{w}$ under $R_{(2 i+1) / 2 n}$.

If it were true that all the $R$ 's agreed, we could use $F$ and an extension of the $R$ 's for $D_{1}$ and $H_{1}$. However, there is no reason why they must agree so we get a chain $D_{1}$ that refines $F$ and a chain map $H_{1}$. In obtaining $H_{1}$ we shall be influenced by $R_{i / n}$ near $p_{i / n}$ and by $R_{(2 i+1) / 2 n}$ on the part of $P$ between $p_{i / n}$ and $p_{(i+1) / n}$.

(c) We mentioned that we are influenced by $R_{i / n}$ near $p_{i / n}$. We now describe the part $P_{i}$ of $P$ near $p_{i / n}$ where we were influenced.

Let $\epsilon$ be a positive number less than $1 / 2 n$ and so small that if $f_{t}, f_{t+1}, \cdots, f_{u}$ is the subchain of $F$ irreducibly covering $p_{i / n}$ and $e_{r}, e_{r+1}, \cdots, e_{8}$ is the image of $f_{t}, f_{t+1}, \cdots, f_{u}$ under $R_{i / n}$, then $f_{t}, f_{t+1}, \cdots, f_{u}$ properly covers each element of $\left\{p_{x}|| x-i / n \mid \leqq \epsilon\right\}$ and $e_{r}, e_{r+1}, \cdots, e_{s}$ properly covers each element of $\left\{q_{x}|| x-i / n \mid \leqq \epsilon\right\}$. We shall describe $P_{i}$ so that $P_{i} \subset P(i / n-\epsilon$, $i / n+\epsilon)$.

Since each of $R_{i / n}, R_{(2 i+1) / 2 n}$, and $R_{(2 i-1) / 2 n}$ takes $f_{t}, f_{t+1}, \cdots, f_{u}$ (and possibly more) onto a subchain (possibly a different one) of $E_{1}$ that properly covers $q_{i / n}$, it follows from Theorem 6 that there are an $f_{y}$ and an $f_{z}$ of $f_{t}$, 
$f_{t+1}, \cdots, f_{u}$ such that $R_{(2 i+1) / n}\left(f_{y}\right), R_{i / n}\left(f_{y}\right)$ are adjacent and $R_{(2 i-1) / 2 n}\left(f_{z}\right)$, $R_{i / n}\left(f_{z}\right)$ are adjacent.

By Theorem 5 there is an open set $U_{i}$ in $P(i / n, i / n+\epsilon) \cdot f_{y}$ such that $\bar{U}_{i} \subset f_{y}$ and $P-U_{i}$ is the sum of two mutually exclusive closed sets $A_{i}, B_{i}$ such that $P(0, i / n) \subset A_{i}, P(i / n+\epsilon, 1) \subset B_{i}$, and for each element $p_{x}$ of $\left\{p_{x} \mid i / n \leqq x \leqq i / n+\epsilon\right\}$, there is one of the sets $p_{x} \cdot A_{i}, p_{x} \cdot B_{i}$ that intersects each element of $f_{t}, f_{t+1}, \cdots, f_{u}$.

Also, there is an open set $V_{i}$ in $P(i / n-\epsilon, i / n)$ such that $\bar{V}_{i} \cdot \bar{U}_{i}=0$, $\bar{V}_{i} \subset f_{z}$, and $P-V_{i}$ is the sum of two mutually exclusive closed sets $A_{i}^{\prime}, B_{i}^{\prime}$ such that $P(0,1 / n-\epsilon) \subset A_{i}^{\prime}, P(i / n, 1) \subset B_{i}^{\prime}$, for each element $p_{x}$ of $\left\{p_{x} \mid i / n-\epsilon \leqq x \leqq i / n\right\}$ there is one of the sets $p_{x} \cdot A_{i}^{\prime}, p_{x} \cdot B_{i}^{\prime}$ that intersects each element of $f_{t}, f_{t+1}, \cdots, f_{u}$.

Then $P_{i}=A_{i} \cdot B_{i}^{\prime}$ if $i=1,2, \cdots, n-1$. If $i=0, P_{i}=A_{i}$; if $i=n, P_{i}=B_{i}^{\prime}$. We note that $p_{i / n} \subset P_{i}$.

(d) In this step we define the chain $D_{1}$. Let $D_{1}$ be a chain irreducibly covering $P$ of mesh so small that (1) if an element $d$ of $D_{1}$ intersects $P_{i}$, it lies in $P_{i}+U_{i}+V_{i}$ and in a link of the subchain of $F$ that irreducibly covers $p_{i / n},(2)$ if $d$ does not intersect any $P_{i}$, then it lies in some $P(i / n,(i+1) / n)$ and in a link of the subchain of $F$ that irreducibly covers $P(i / n,(i+1) / n)$, (3) $d$ does not intersect both $U_{i}$ and $V_{i}$ but it lies in $f_{\nu}$ or $f_{z}$ according as it intersects $U_{i}$ or $V_{i}$. We note that if $d$ intersects $P_{i}$, it lies in an element of $F$ on which $R_{i / n}$ is defined and otherwise it lies in an element of $F$ on which some $R_{(2 i+1) / 2 n}$ is defined.

(e) We now describe the chain map $H_{1}$ of $D_{1}$ onto $E_{1}$. Suppose $d$ is an element of $D_{1}$ that lies in $f_{k}$ of $F$. If $d$ intersects the $U_{i}$ mentioned in Step 2c, then we pick $f_{k}$ to be $f_{y}$; then $H_{1}(d)$ is $R_{i / n}\left(f_{y}\right)$ or $R_{(2 i+1) / 2 n}\left(f_{y}\right)$ according as $d$ does or does not intersect $P_{i}$. If $d$ intersects $V_{i}$, then we pick $f_{k}$ to be $f_{z}$; $H_{1}(d)$ is $R_{i / n}\left(f_{z}\right)$ or $R_{(2 i-1) / 2 n}\left(f_{z}\right)$ according as $d$ does or does not intersects $P_{i}$. If $d$ intersects $P_{i}$ but neither $U_{i}$ nor $V_{i}$, pick $f_{k}$ to be any link of $F$ on which $R_{i / n}$ is defined; then $H_{1}(d)=R_{i / n}\left(f_{k}\right)$. If $d$ does not intersect any $V_{i}+P_{i}+U_{i}$, it lies in some $P(i / n,(i+1) / n)$ so pick $f_{k}$ to be some link of $F$ on which $R_{(2 i+1) / 2 n}\left(f_{k}\right)$ is defined; then $H_{1}(d)=R_{(2 i+1) / 2 n}\left(f_{k}\right)$.

(f) Here we show that $H_{1}$ is a chain map-that is $H_{1}\left(d_{j}\right)$ is adjacent to $H_{1}\left(d_{j+1}\right)$ where $d_{j}, d_{j+1}$ are adjacent elements of $D_{1}$. If both $d_{j}$ and $d_{j+1}$ intersect $P_{i}$, then $H_{1}\left(d_{j}\right)$ is adjacent to $H_{1}\left(d_{j+1}\right)$ because $R_{i / n}$ is a chain map. If one intersects $P_{i}$ and the other does not, either both intersect $U_{i}$ or both intersect $V_{i}$. If both intersect $U_{i}$, both lie in $f_{y}$ and $R_{i / n}\left(f_{y}\right)$ is adjacent to $R_{(2 i+1) / 2 n}\left(f_{y}\right)$; if both intersect $V_{i}$, both lie in $f_{z}$ and $R_{i / n}\left(f_{z}\right)$ is adjacent to $R_{(2 i-1) / 2 n}\left(f_{z}\right)$. If neither $d_{j}$ nor $d_{j+1}$ intersect any $P_{i}$, then $H_{1}\left(d_{j}\right)$ is adjacent to $H_{1}\left(d_{j+1}\right)$ because each $R_{(2 i+1) / 2 n}$ is a chain map.

(g) In Step $2 \mathrm{~g}$ we show that the chain map $H_{1}$ satisfies Condition I mentioned at the beginning of Step 2.

Suppose $d_{j}$ intersects $p_{i / n}, d_{j} \subset f_{k}$, and $H_{1}\left(d_{j}\right)=R_{i / n}\left(f_{k}\right)$. We find from the 
definition of $R_{i / n}$ in Step $2 \mathrm{~b}$ that $h\left(f_{k} \cdot p_{i / n}\right) \subset R_{i / n}\left(f_{k}\right)$. Hence $h\left(d_{j} \cdot p_{i / n}\right)$ $\subset R_{i / n}\left(f_{k}\right)=H_{1}\left(d_{j}\right)$.

(h) Now we turn to Condition II. Suppose that $d_{j}$ intersects $p_{x}(i / n \leqq x$ $\leqq(i+1) / n)$. First we show that $H_{1}\left(d_{j}\right)$ intersects $q_{x}$.

There is an element $f_{k}$ of $F$ containing $d_{j}$ such that $H_{1}\left(d_{j}\right)$ is equal to either $R_{i / n}\left(f_{k}\right), R_{(2 i+1) / 2 n}\left(f_{k}\right)$, or $R_{(i+1) / n}\left(f_{k}\right)$. If $H_{1}\left(d_{j}\right)=R_{i / n}\left(f_{k}\right), H_{1}\left(d_{j}\right)$ is a link of the subchain of $E_{1}$ irreducibly covering $q_{i / n}$ and hence intersects $q_{x}$ as a result of conditions in Step $1 \mathrm{~b}$; if $H_{1}\left(d_{j}\right)=R_{(i+1) / n}\left(f_{k}\right), H_{1}\left(d_{j}\right)$ is a link of the subchain of $E_{1}$ irreducibly covering $q_{(i+1) / n}$ and hence intersects $q_{x}$; if $H_{1}\left(d_{j}\right)$ $=R_{(2 i+1) / 2 n}\left(f_{k}\right), H_{1}\left(d_{j}\right)$ is a link of the subchain of $E_{1}$ irreducibly covering $Q(i / n,(i+1) / n)$ as noted in Step $2 \mathrm{~b}$ and hence intersects each element of $\left\{q_{x} / i / n \leqq x \leqq(i+1) / n\right\}$ by Step $1 \mathrm{~b}$.

Suppose $d_{v}, d_{v+1}, \cdots, d_{w}$ is the subchain of $D_{1}$ that irreducibly covers $p_{x}$. Now we show that if $e_{k}$ is an element of the subchain of $E_{1}$ irreducibly covering $q_{x}$, then there is an element $d_{j}$ of $d_{v}, d_{v+1}, \cdots, d_{w}$ such that $e_{k}=H_{1}\left(d_{j}\right)$.

If $p_{x}$ intersects $P_{i}+U_{i}$ it follows from the definition of $\epsilon$ in Step 2c that $e_{k}$ is an element of the subchain $e_{r}, e_{r+1}, \cdots, e_{s}$ of $E_{1}$ that irreducibly covers $q_{i / n}$.

If $p_{x} \cdot P_{i}$ intersects each element of the subchain $f_{t}, f_{t+1}, \cdots, f_{u}$ of $F$ that irreducibly covers $p_{i / n}$, consider the three consecutive elements $f_{a-1}, f_{a}, f_{a+1}$ of $f_{t}, f_{t+1}, \cdots, f_{u}$ that go into $e_{k}$ under $R_{i / n}$ as mentioned in Step 2b. There is a $d_{j}$ of $d_{v}, d_{v+1}, \cdots, d_{w}$ such that $d_{j} \cdot p_{x} \cdot P_{i}$ intersects $f_{a}$. Then $H_{1}\left(d_{j}\right)$ is equal to either $R_{i / n}\left(f_{a-1}\right), R_{i / n}\left(f_{a}\right)$, or $R_{i / n}\left(f_{a+1}\right)$ and all three of them are equal to $e_{k}$.

In case $p_{x}$ intersects $P_{i}+U_{i}$ but $p_{x} \cdot P_{i}$ does not intersect each of $f_{l}$, $f_{t+1}, \cdots, f_{u}$, then $p_{x}-\left(U_{i}+P_{i}\right)$ intersects each element of $f_{t}, f_{t+1}, \cdots, f_{u}$ by definition of $A_{i}, B_{i}$ in Step 2c. Now let $f_{a-1}, f_{a}, f_{a+1}$ be three consecutive elements of the subchain $f_{t}, f_{t+1}, \cdots, f_{u}$ that go into $e_{k}$ under $R_{(2 i+1) / 2 n}$. There is a $d_{j}$ of $d_{v}, d_{v+1}, \cdots, d_{w}$ such that $d_{j}$ lies in $f_{a}$ and intersects $p_{x}-\left(U_{i}+P_{i}\right)$. Then $H_{1}\left(d_{j}\right)$ is either $R_{(2 i+1) / 2 n}\left(f_{a-1}\right), R_{(2 i+1) / 2 n}\left(f_{a}\right)$, or $R_{(2 i+1) / 2 n}\left(f_{a+1}\right)$ and all three are equal to $e_{k}$.

In case $p_{x}$ intersects $V_{i+1}+P_{i+1}$, we also find that there is an element $d_{j}$ of $d_{v}, d_{v+1}, \cdots, d_{w}$ such that $e_{k}=H_{1}\left(d_{j}\right)$.

In case $p_{x}$ does not intersect $P_{i}+U_{i}+V_{i+1}+P_{i+1}$, we find that $e_{k}$ is a link of the subchain of $E_{k}$ that irreducibly covers $Q(i / n,(i+1) / n)$. Let $f_{t}, f_{t+1}, \cdots, f_{u}$ be the subchain of $F$ that irreducibly covers $p_{x}$ and $f_{a-1}, f_{a}$, $f_{a+1}$ be the three consecutive elements of $f_{t}, f_{t+1}, \cdots, f_{u}$ that go into $e_{k}$ under $R_{(2 i+1) / 2 n}$. Again we find that there is an element $d_{j}$ of $d_{v}, d_{v+1}, \cdots, d_{w}$ in $f_{a}$ such that $H_{1}\left(d_{j}\right)=e_{k}$.

(i) As in Step $1 \mathrm{~b}$ we find that there is an integer $k$ such that $k$ is a multiple of $n$ and if $0 \leqq b-a \leqq 1 / k$, the subchain of $D_{1}$ that irreducibly covers $P(a, b)$ properly covers each element of $\left\{p_{x} \mid a \leqq x \leqq b\right\}$ and the image of this subchain under $H_{1}$ properly covers each element of $\left\{q_{x} \mid a \leqq x \leqq b\right\}$. 
We now extend the homeomorphism $h$ to $p_{0}, p_{1 / k}, p_{2 / k}, \cdots, p_{1}$. This is done so that $h\left(p_{i / k}\right)=q_{i / k}$ and such that for each element $d_{j}$ of the subchain of $D_{1}$ that properly covers $p_{i / k}, h\left(p_{i / k} \cdot d_{j}\right) \subset H_{1}\left(d_{j}\right)$. Theorem 9 assures us that $h$ can be extended in this fashion.

Step 3. In this step we define a chain $E_{2}$ covering $Q$, a chain map $K_{1}$ of $E_{2}$ onto $D_{1}$, and extend the map $h$ to additional elements of $\left\{p_{x}\right\}$. The step is much like Step 2. We are getting an approximation to $h^{-1}$ instead of to $h$.

We show that there is a $1 / 4$-chain $E_{2}$ irreducibly covering $Q$ and a chain map $K_{1}$ of $E_{2}$ onto $D_{1}$ such that

I. If link $e_{i}$ of $E_{2}$ intersects $q_{j / k}$, then $h^{-1}\left(e_{i} \cdot q_{j / k}\right) \subset K_{1}\left(e_{i}\right)$.

II. For each $x, K_{1}$ takes any subchain of $E_{2}$ properly covering $q_{x}$ onto a subchain of $D_{1}$ properly covering $p_{x}$.

III. $e_{i} \subset H_{1} K_{1}\left(e_{i}\right)$ for each link $e_{i}$ of $E_{2}$.

The thing making Step 3 more complicated than Step 2 is Condition III. The proof that there are $E_{2}$ and $K_{1}$ satisfying Conditions I and II is similar to that showing that there are $D_{1}$ and $H_{1}$ satisfying Conditions I and II of Step 2.

Here we show how to alter the arguments of Step 2 to get $E_{2}$ and $K_{1}$ to satisfy Condition III in addition to I and II.

As in Step 2, we get an approximation $F$ to $E_{2}$. The $F$ of Step 3 will be an $\epsilon$-chain irreducibly covering $Q$ where $\epsilon$ is small enough to make the following statements true. (1) $\epsilon<1 / 4$. (2) No link of $F$ intesects $q_{a}$ and $q_{b}$ if $1 / 2 k<b$ $-a$. (3) The image under $h^{-1}$ of any subset of $q_{0}+q_{1 / k}+\cdots+q_{1}$ of diameter less than $5 \epsilon$ lies in an element of $E_{1} .(4) \epsilon$ is less than the $\epsilon$ mentioned in the corollary to Theorem 8 where the $D(r, s)$ there is the subchain of $D$ irreducibly covering $P(i / k,(i+1) / k)(i=0,1, \cdots, k-1)$ and $E$ is the image of $D(r, s)$ under $H_{1}$.

The chain map $R_{i / k}$ we define here is similar to the $R_{i / n}$ 's defined in Step $2 \mathrm{~b}$ in that $R_{i / k}$ takes the subchain $f_{t}, f_{t+1}, \cdots, f_{u}$ of $F$ that irreducibly covers $q_{i / k}$ on to the subchain $d_{v}, d_{v+1}, \cdots, d_{w}$ of $D_{1}$ that irreducibly covers $p_{i / k}$ such that each element of $d_{v}, d_{v+1}, \cdots, d_{v}$ is the image of three consecutive elements of $f_{t}, f_{t+1}, \cdots, f_{u}$ and for each $j, h^{-1}\left(q_{i / k} \cdot f_{j}\right) \subset R_{i / k}\left(f_{j}\right)$. Operating on both sides of $h^{-1}\left(q_{i / k} \cdot f_{j}\right) \subset p_{i / k} \cdot R_{i / k}\left(f_{j}\right)$ by $h$ we find that $q_{i / k} \cdot f_{j} \subset h\left(p_{i / k}\right.$ - $\left.R_{i / k}\left(f_{j}\right)\right)$. Since $h\left(p_{i / k} \cdot R_{i / k}\left(f_{j}\right)\right) \subset H_{1} R_{i / k}\left(f_{j}\right)$ by Step $2 \mathrm{i}$, we have that $q_{i / k}$ $\cdot f_{j} \subset H_{1} R_{i / k}\left(f_{j}\right)$.

To complete the analogue of Step $2 \mathrm{~b}$, we only need to pick a chain map $R_{(2 i+1) / 2 k}$. It takes the subchain $f_{t}, f_{i+1}, \cdots, f_{u}$ of $F$ that irreducibly covers $Q(i / k,(i+1) / k)$ onto the subchain $d_{r}, d_{r+1}, \cdots, d_{s}$ of $D_{1}$ that irreducibly covers $P(i / k,(i+1) / k)$ so that (1) $f_{j} \subset H_{1} R_{(2 i+1) / 2 k}\left(f_{j}\right)$ and (2) for each $x$ $(i / k \leqq x \leqq(i+1) / k), R_{(2 i+1) / 2 k}$ takes the subchain $f_{v}, f_{v+1}, \cdots, f_{w}$ of $F$ irreducibly covering $q_{x}$ onto $d_{r}, d_{r+1}, \cdots, d_{s}$ so that each element of $d_{r}$, $d_{r+1}, \cdots, d_{s}$ is the image of three consecutive links of $f_{v}, f_{v+1}, \cdots, f_{w}$. That there is such a map $R_{(2 i+1) / 2 k}$ follows from the fact that the mesh of the chain 
$F$ was taken smaller than the $\epsilon$ mentioned in the corollary to Theorem 8 .

In the analogue to Step 2c we pick $\epsilon$ so small that the $Q_{i}, V_{i}, U_{i}$ we obtain will have the property that if $f_{j}$ is an element of the subchain of $F$ irreducibly covering $q_{i / k}$, then $\left(V_{i}+Q_{i}+U_{i}\right) \cdot f_{j} \subset H_{1} R_{i / k}\left(f_{j}\right)$. (We already have that $q_{i / k} \cdot f_{j} \subset H_{1} R_{i / k}\left(f_{j}\right)$ from the definition of $R_{i / k}$.)

Now the argument in Step 3 proceeds as the argument in Step 2 through parts $\mathrm{c}, \mathrm{d}, \mathrm{e}, \mathrm{f}, \mathrm{g}, \mathrm{h}$, and $\mathrm{i}$. The purpose in extending the homeomorphism $h$ in part (i) is to help in setting up Step 4. We complete the discussion of Step 3 by showing that the $E_{2}$ we obtained in the analogue of Step 2d satisfies Condition III with respect to the $K_{1}$ we obtained in the analogue of Step 2e.

Suppose the link $e_{j}$ of $E_{2}$ intersects $Q_{i}$ (where $Q_{i}$ is the analogue of $P_{i}$ in Step 2c), $e_{j} \subset f_{z}$, and $K_{1}\left(f_{z}\right)=R_{i / k}\left(f_{z}\right)$. Then $f_{z}$ is a link of the subchain of $F$ irreducibly covering $q_{i / k}$ and $e_{j} \subset V_{i}+Q_{i}+U_{i}$. Since $\left(V_{i}+Q_{i}+U_{i}\right)$ $\cdot f_{z} \subset H_{1} R_{i / k}\left(f_{z}\right), e_{j} \subset H_{1} K_{1}\left(e_{j}\right)$.

Suppose $e_{j}$ does not intersect any $Q_{i}$. Then there is an $f_{z}$ such that $e_{j} \subset f_{z}$ and $K_{1}\left(e_{j}\right)=R_{(2 i+1) / 2 k}\left(f_{z}\right)$. But since $f_{z} \subset H_{1} R_{(2 i+1) / 2 k}\left(f_{z}\right), e_{j} \subset H_{1} K_{1}\left(e_{j}\right)$.

Steps $4,5, \cdots$. These steps are essentially repetitions of Step 3. We have Conditions $\mathrm{A}, \mathrm{A}^{\prime}, \mathrm{B}, \mathrm{B}^{\prime}$ satisfied which are modifications of Conditions II and III of Step 3.

In Step $2 \mathrm{n}$ we find a chain $D_{n}$ irreducibly covering $P$ and a chain map $H_{n}$ of $D_{n}$ onto $E_{n}$ such that the following conditions hold.

A. For each $x(0 \leqq x \leqq 1), H_{n}$ takes any subchain of $D_{n}$ properly covering $p_{x}$ onto a subchain of $E_{n}$ properly covering $q_{x}$.

B. $d_{i} \subset K_{n-1} H_{n}\left(d_{i}\right)$ for each link $d_{i}$ of $D_{n}$.

In Step $2 n+1$ we get a chain $E_{n+1}$ irreducibly covering $Q$ and a chain map $K_{n}$ of $E_{n+1}$ onto $D_{n}$ satisfying the following conditions.

$\mathrm{A}^{\prime}$. For each $x(0 \leqq x \leqq 1), K_{n}$ takes any subchain of $E_{n+1}$ properly covering $q_{x}$ onto a subchain of $D_{n}$ properly covering $p_{x}$.

$\mathrm{B}^{\prime} . e_{i} \subset H_{n} K_{n}\left(e_{i}\right)$ for each link $e_{i}$ of $E_{n+1}$.

Although we carry along analogues of Condition I of Step 3, the only part of this that will interest us henceforward is the following.

C. If link $d_{i}$ of $D_{n}$ intersects $p_{0}+p_{1}$, then $h\left(d_{i} \cdot\left(p_{0}+p_{1}\right)\right) \subset H_{n}\left(d_{i}\right)$.

$\mathrm{C}^{\prime}$. If link $e_{i}$ of $E_{n+1}$ intersects $q_{0}+q_{1}$, then $h^{-1}\left(e_{i} \cdot\left(q_{0}+q_{1}\right)\right) \subset K_{n}\left(e_{i}\right)$.

Definition of HOMEOMORPHISM $h$. For each point $p$ of $P$ let $d(p, i)$ denote the sum of the elements of $D_{i}$ containing $p$ and $H_{i} d(p, i)$ denote the sum of the images under $H_{i}$ of the elements of $D_{i}$ in $d(p, i)$. We note that $d(p, i)$ is the sum of one or two adjacent links of $D_{i}$ while $H_{i} d(p, i)$ is the sum of one or two adjacent links of $E_{i}$.

Of course, $d(p, i+1) \subset d(p, i)$. We now show that $I_{i+1} d(p, i+1) \subset H_{i} d(p, i)$ by showing that if $e_{j}^{i+1}=H_{i+1}\left(d_{k}^{i+1}\right)$ where $d_{k}^{i+1}$ is an element of $D_{i+1}$ in $d(p, i+1)$, then $e_{j}^{i+1} \subset H_{i} d(p, i)$. Since $d_{k}^{i+1} \subset K_{i} H_{i+1}\left(d_{k}^{i+1}\right)$ by Condition $\mathrm{B}$, the link $K_{i} H_{i+1}\left(d_{k}^{i+1}\right)=K_{i}\left(e_{j}^{i+1}\right)$ of $D_{i}$ lies in $d(p, i)$. Also, $e_{j}^{i+1} \subset H_{i} K_{i}\left(e_{j}^{i+1}\right)$ by Condition $\mathrm{B}^{\prime}$ and $H_{i} K_{i}\left(e_{j}^{i+1}\right)=H_{i}\left(d_{k}^{i+1}\right) \subset H_{i} d(p, i)$. 
For each point $p$ of $P$, let $h(p)$ be the intersection of the closures of the decreasing sequence of open sets $H_{1}(d(p, 1)), H_{2}(d(p, 2)), H_{3}(d(p, 3)), \cdots$. The intersection exists because $\left.H_{i+1} d(p, i+1)\right) \subset H_{i}(d(p, i))$. It is a point because the diameter of the closure of $H_{i}(d(p, i))$ is less than $2 / 2^{i}$.

If $q$ is any point of $d(p, i)$, the diameter of $H_{i}(d(p, i))+H_{i}(d(q, i))$ is less than $3 / 2^{i}$ so $\rho(h(p), h(q))<3 / 2^{i}$. Therefore $h$ is continuous.

If $p \in p_{x}$, we find from Condition $\mathrm{A}$ that $H_{i}(d(p, i))$ intersects $q_{x}$. Hence $h(p) \in q_{x}$.

We now show that $h$ takes $P$ onto $Q$. Let $q$ be a point of $Q$ and $e_{j}^{i}$ be an element of $E_{i}$ containing $q$. There is an element $d_{k}^{i}$ of $D_{i}$ such that $H_{i}\left(d_{k}^{i}\right)=e_{j}^{i}$. Therefore for some point $p$ of $P, e_{j}^{i} \subset H_{i}(d(p, i))$. Since the diameter of $H_{i}(d(p, i))$ is less than $2 / 2^{i}, \rho(q, h(p))<2 / 2^{i}$. This shows that $h(P)$ is dense in $Q$. Since $h(P)$ is closed, it is equal to $Q$.

The transformation $h$ we have defined agrees with the given homeomorphism $h$ on $p_{0}+p_{1}$ because for each point $p$ of $p_{0}+p_{1}, H_{i}(d(p, i))$ contains $h(p)$ by Condition C.

Finally we show that $h$ is $1-1$. Suppose $h(p)=h(q)$. Since the closures of $H_{i} d(p, i)$ and $H_{i} d(q, i)$ intersect and we are dealing in this paper only with chains whose nonadjacent links do not have closures that intersect, then $H_{i} d(p, i)$ intersects $H_{i} d(q, i)$. Since $K_{i-1}$ is a chain map, the set $K_{i-1} H_{i} d(p, i)$ intersects the set $K_{i-1} H_{i} d(q, i)$. But the closures of these two sets contain $p$ and $q$ respectively so $\rho(p, q)<4 / 2^{i}$. Therefore $p=q$ if $h(p)=h(q)$.

7. A circle of pseudo-arcs. It follows from Theorem 10 that each circle of pseudo-arcs is homogeneous and that any two of them are homeomorphic. In this section we show that the plane contains a circle of pseudo-arcs. But we first describe an analogous upper-semicontinuous collection $G$ of arcs in the plane.

Let $W_{1}$ and $W_{2}$ be circles in the plane with center at $(0,0)$ and radii equal to one and two, respectively. We shall define a collection $\left\{g_{x} \mid-\pi \leqq x \leqq \pi\right\}$ of mutually exclusive arcs such that each $g_{x}$ is a straight line interval which is irreducible from $W_{1}$ to $W_{2}$ (or which is the sum of two such straight line intervals whose intersection is a common end point), $g_{-\pi}=g_{\pi}$, and $\sum g_{x}(-\pi \leqq x \leqq \pi)$ is a circle-like continuum.

Let $g_{-\pi}$ be the sum of the two straight line intervals from $(r=1, \theta=-\pi)$ to $(r=2, \theta=-\pi \pm \pi / 12)$. Let $g_{\pi}=g_{-\pi}$. Let $g_{0}$ be the sum of the two straight line intervals from $(1,0)$ to $(2, \pm \pi / 12)$. Let $g_{\pi / 2}$ be the sum of the straight line intervals from $(2, \pi / 2)$ to $(1, \pi / 2 \pm \pi / 12)$ and let $g_{-\pi / 2}$ be the sum of the straight line intervals from $(2,-\pi / 2)$ to $(1,-\pi / 2 \pm \pi / 12)$. Because of their shape, these elements of $\left\{g_{x}\right\}$ will be called $V$ 's.

Now let $U$ denote the set of all points of the annulus $W_{1} W_{2}$ not belonging to any $V$ defined thus far. There are a finite number of components of $U$ which intersect both $W_{1}$ and $W_{2}$ and in each of these components two $V$ 's will be defined. Let the arc $T_{i}(i=1,2)$ be the closure of the intersection of 
such a component of $U$ with $W_{i}$. One end point of $T_{i}(i=1,2)$ is an end point of a $V$ and the other end point of $T_{i}$ is the vertex of a $V$. Now construct two disjoint $V$ 's in $U$ such that one of these $V$ 's has its end points on $T_{i}$ $(i=1,2)$, the other has its vertex on $T_{i}$ and these three points are the quarter points of $T_{i}$. The reader should note that on $W_{1}$ or $W_{2}$ no vertex of a $V$ is adjacent to a vertex of a $V$ and no end point of a $V$ is adjacent to an end point of another $V$.

After the above construction of a pair of $V$ 's has been carried out for every component of $U$ which intersects both $W_{1}$ and $W_{2}$, a new $U$ may be defined and the process continued countably many $\left(\boldsymbol{\aleph}_{0}\right)$ times. Let $G$ denote the collection of all the $V$ 's together with each straight line interval which is the limit of a convergent sequence of $V$ 's but which is not a subset of a $V$. By appropriately choosing the subscripts $x,\left\{g_{x} \mid-\pi \leqq x \leqq \pi\right\}$ is the collection $G$. It is easy to see that $G$ is an upper-semicontinuous collection of arcs, $G$ is a circle (if its elements are thought of as points) and $G^{*}$ (the sum of the elements of $G$ ) is a circle-like continuum. Something quite similar to the fact that $G^{*}$ is circle-like has been previously observed by Roberts [25].

The reader will note that $G$ is not a continuous collection. This is due fundamentally to the fact that in the plane an arc has two sides. Furthermore $G^{*}$ is not homogeneous for still another reason, namely, some points of $G$ are local separating points (the vertices of the $V$ 's) while others are not. But if pseudo-arcs are substituted for the $V^{\prime}$ 's, it should be possible to eliminate these two properties from $G$ and $G^{*}$ respectively while in general keeping $G$ and $G^{*}$ unchanged in other respects.

As before let $W_{1}$ and $W_{2}$ be concentric circles in the plane with center at $(0,0)$ and radii one and two, respectively. Since there are countably many $V$ 's of the preceding construction, let them be $V_{1}, V_{2}, V_{3}, \cdots$ in inverse order to the polar angle between $a_{i}$ and $c_{i}$ (the end points of $V_{i}$ ). Let $b_{i}$ be the vertex of $V_{i}$ and for convenience let $b_{i}$ belong to $W_{1}$ when $i$ is odd but belong to $W_{2}$ when $i$ is even.

Now there exists a sequence $D_{1}, D_{2}, D_{3}, \cdots$ of circular chains of simple domains in the plane such that

(1) for each positive integer $i$, the closure of each element of $D_{i+1}$ is a subset of some element of $D_{i}$;

(2) for each $i$, each element of $D_{i}$ intersects the annulus $W_{1} W_{2}$ and not both of two intersecting links of $D_{i}$ intersect $W_{1}+W_{2}$,

(3) if (for each $i$ ) $\delta_{i}$ is the maximum diameter of a link of $D_{i}$, then $\delta_{i} \rightarrow 0$,

(4) the subscripts of the elements of $D_{i}$ which intersect $W_{1}$ preserve the counterclockwise order on $W_{1}$ and the subscripts of these intersecting $W_{2}$ preserve the counterclockwise order on $W_{2}$,

(5) if $a_{i}, b_{i}$, and $c_{i}$ are the end points and vertex of $V_{i}$, there is a natural number $m(i)$ such that the (shortest) subchain of $D_{m(i)}$ irreducible from $a_{i}$ to $c_{i}$ contains $b_{i}$, the subchain of $D_{m(i)+1}$ irreducible from $a_{i}$ to $b_{i}$ contains $c_{i}$, 
the subchain of $D_{m(i)+2}$ irreducible from $b_{i}$ to $c_{i}$ contains $a_{i}$, the subchain of $D_{m(i)+3}$ irreducible from $a_{i}$ to $c_{i}$ contains $b_{i}$, etc.,

(6) $\left(W_{1}+W_{2}\right) \cdot \Pi D_{i}^{*}=$ closure of $\sum\left(a_{i}+b_{i}+c_{i}\right)$ as in the preceding example,

(7) for each $i, D_{i}$ is the sum of finitely many subchains $T_{i 1}, T_{i 2}, \cdots$, $T_{i n(i)}$ such that (a) $T_{i 1}^{*}, T_{i 2}^{*}, \cdots, T_{i n(i)}^{*}$ is a circular chain, and (b) for each $j$, $[1 \leqq j \leqq n(i)], T_{i j}$ is either irreducible from $W_{1}$ to $W_{2}$ or (for some $k$ ) irreducible about $a_{k}+b_{k}+c_{k}$, and

(8) if $h<i$, each element of $\left\{T_{i}^{*}\right\}_{j=1}^{n(1)}$ is a subset of two intersecting elements of $\left\{T_{h j}^{*}\right\}_{j=1}^{n(h)}$ and

(9) if $h<i$ and $T_{i j}$ is a refinement of $T_{h k}+T_{h l}, T_{i j}$ is crooked [3] with respect to $T_{h k}+T_{h l}$ where $l=(k+1) \bmod n(h)$.

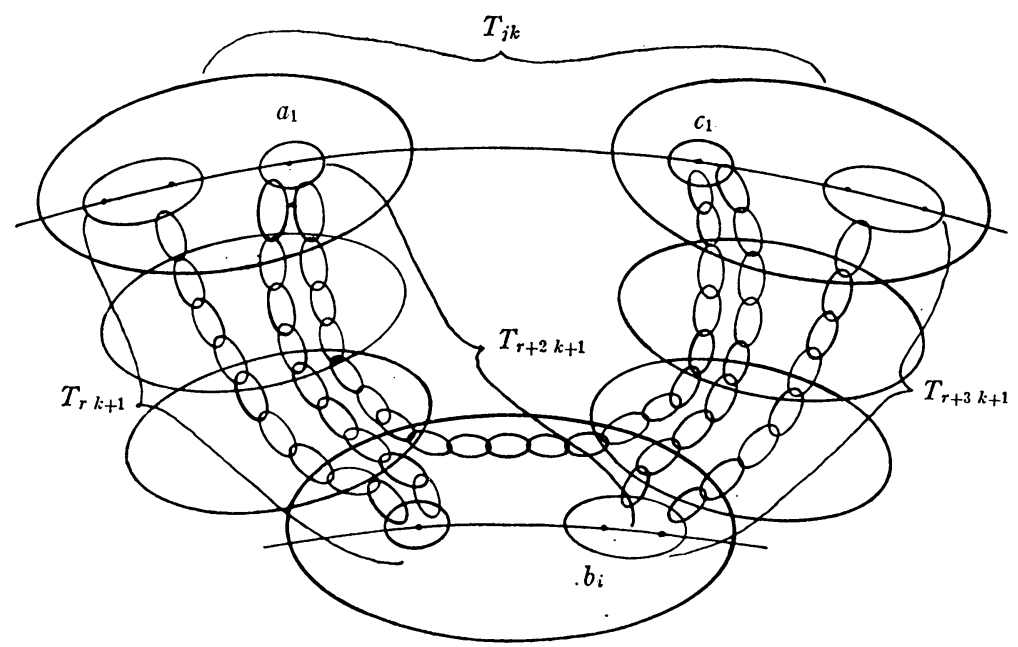

FIG. 3

Now let $M=\Pi D_{i}^{*}$ and let $G$ denote the set of all subcontinua of $M$ which are irreducible from $W_{1}$ to $W_{2}$. The reader will observe that if (9) were omitted the construction of the chains $D_{i}$ could be carried out in such a way that each element of $G$ would be an arc if its intersection with $W_{1}+W_{2}$ were two points and indecomposable if this intersection were three points. Also, in this case $M$ would be a continuous circle-like circle of continua. With the addition of (9) one may see with the help of [3] that each element of $G$ is a pseudo-arc. It follows from Theorem 10 that $M$ is homogeneous.

\section{BIBLIOGRAPHY}

1. R. D. Anderson, On monotone interior mappings in the plane, Trans. Amer. Math. Soc. vol. 73 (1952) pp. 211-222. 
2. - Report on results on continuous collections of pseudo-arcs in the plane and homogeneity of the universal curve, Summary of Lectures and Seminars, Summer Institute on Set Theoretic Topology, Madison, 1955, pp. 76-77.

3. R. H. Bing, A homogeneous indecomposable plane continuum, Duke Math. J. vol. 15 (1948) pp. 729-742.

4. - Concerning hereditarily indecomposable continua, Pacific J. Math. vol. 1 (1951) pp. $43-51$.

5. —-, Snake-like continua, Duke Math. J. vol. 18 (1951) pp. 653-663. 766.

6. - Another homogeneous plane continuum, Bull. Amer. Math. Soc. Abstract 60-6-

7. - The pseudo-arc, Summary of Lectures and Seminars, Summer Institute on Set Theoretic Topology, Madison, 1955, pp. 70-73.

8. C. E. Burgess, Some theorems on n-homogeneous continua, Proc. Amer. Math. Soc. vol. 5 (1954) pp. 136-143.

9. - Certain types of homogeneous continua, Proc. Amer. Math. Soc. vol. 6 (1955) pp. 348-350.

10. - Homogeneous continua, Summary of Lectures and Seminars, Summer Institute on Set Theoretic Topology, Madison, 1955, pp. 73-76.

11. Gustav Choquet, Prolongements d'homéomorphies. Ensembles topologiquement nommables, Caractérization topologique individuelle des ensembles fermés totalement discontinus, C.R. Acad. Sci. Paris, vol. 219 (1944) pp. 542-544.

12. H. J. Cohen, Some results concerning homogeneous plane continua, Duke Math. J. vol. 18 (1951) pp. $467-474$.

13. F. B. Jones, $A$ note on homogeneous plane continua, Bull. Amer. Math. Soc. vol. 55 (1949) pp. 113-114.

14. - Certain homogeneous unicoherent indecomposable continua, Proc. Amer. Math. Soc. vol. 2 (1951) pp. 855-859.

15. - On a certain type of homogeneous plane continuum, Bull. Amer. Math. Soc. Abstract 60-6-770.

16. ——, On homogeneity, Summary of Lectures and Seminars, Summer Institute on Set Theoretic Topology, Madison, 1955, pp. 66-68.

17. - On a certain type of homogeneous plane continuum, Proc. Amer. Math. Soc. vol. 6 (1955) pp. 735-740.

18. I. Kapuano, Sur une proposition de M. Bing, C. R. Acad. Sci. Paris vol. 236 (1953) pp. 2468-2469.

19. - Sur les continus linéaires, C. R. Acad. Sci. Paris, vol. 237 (1953) pp. 683-685.

20. B. Knaster, Un continu dont tout sous-continu est indécomposable, Fund. Math. vol. 3 (1922) pp. 247-286.

21. B. Knaster and C. Kuratowski, Problème 2, Fund. Math. vol. 1 (1920) p. 223.

22. S. Mazurkiewicz, Sur les continus homogènes, Fund. Math. vol. 5 (1924) pp. 137-146.

23. E. E. Moise, An indecomposable plane continuum which is homeomorphic to each of its nondegenerate subcontinua, Trans. Amer. Math. Soc. vol. 63 (1948) pp. 581-594.

24. ——, A note on the pseudo-arc, Trans. Amer. Math. Soc. vol. 64 (1949) pp. 57-58.

25. J. H. Roberts, Collections filling a plane, Duke Math. J. vol. 2 (1936) pp. 10-19.

26. Z. Waraszkiewicz, Sur les courbes planes topologiquement homogenes, C. R. Acad. Sci. Paris, vol. 204 (1937) pp. 1388-1390.

The University of Wisconsin, Madison, Wis.

The University of North Carolina,

Chapel Hill, N. C. 